$\mathrm{PM} / 97-26$

GDR-S-002

\title{
Novel Electroweak Symmetry Breaking Conditions From Quantum Effects In The MSSM
}

\author{
Christophe LE MOUËL and Gilbert MOULTAKA目 \\ Physique Mathématique et Théorique \\ UMR-CNRS, \\ Université Montpellier II, F34095 Montpellier Cedex 5, France
}

\begin{abstract}
We present, in the context of the Minimal Supersymmetric Standard Model, a detailed one-loop analytic study of the minimization conditions of the effective potential in the Higgs sector. Special emphasis is put on the role played by $\operatorname{Str} M^{4}$ in the determination of the electroweak symmetry breaking conditions, where first and second order derivatives of the effective potential are systematically taken into account. Novel, necessary (and sufficient in the Higgs sector) model-independent constraints, are thus obtained analytically, leading to new theoretical lower and upper bounds on $\tan \beta$. Although fully model-independent, these bounds are found to be much more restrictive than the existing model-dependent ones! A first illustration is given in the context of a SUGRA-GUT motivated scenario.
\end{abstract}

PACS: 10., 11.30.Qc, 12.38.Bx, 12.60.Jv

Keywords: MSSM, Electroweak symmetry breaking, effective potential, stability, modelindependence, $\tan \beta$.

\footnotetext{
${ }^{1}$ supported in part by EC contract CHRX-CT94-0579

${ }^{2}$ Postal address as above. E-mail: moultaka@lpm.univ-montp2.fr, phone: (33) 04.67.14.35.53,
} fax: (33) 04.67.54.48.50 


\section{Introduction}

Unraveling the origin of the electroweak symmetry breaking (EWSB) remains so far a piece of theoretical challenge, and justifies a wealth of experimental endeavor. Even though (in a "theory-of-everything" state of mind) one assumes that it is ultimately the dynamics at tremendously high energy scales (GUT or Planck or even higher) that should account for the fact that the electroweak vacuum is not (gauge) symmetric, a central question remains:

how would this dynamics effectively translate, over tens of orders of magnitude, down to the electroweak scale?

Phenomenological models of supersymmetry offer plausible scenarios for EWSB [1] triggered by supersymmetry breaking, the latter being presumably connected with dynamics at much higher scales [2], [3]. As is well-known, this radiative EWSB [1] is made possible thanks to the heaviness of the top quark whose experimental discovery around $m_{t} \sim 180 \mathrm{GeV}$ revived the interest in such models. Nonetheless, the breaking of the electroweak symmetry is not a matter of fact in these scenarios, in that one has still to enforce phenomenologically a bounded from below potential and the absence of charge or color breaking minima [四]. This is clear indication that the scheme is not yet dynamically complete, and one might ask whether a mechanism can be found which would naturally "lock" the spontaneous symmetry breaking in the electroweak sector of the theory, at the relevant energy scale. The answer to this is obviously related to the general question asked above, and is out of the scope of the present paper. It will constitute, however, a general motivation for our study. Indeed, an important prerequisite to all this is to improve our understanding of the structure of the effective potential of the Minimal Supersymmetric Standard Model (MSSM) and with it the sufficiency and necessity of the phenomenological conditions for radiative EWSB. One would like to assess as much as possible the implications of the softly broken supersymmetry on the structure of the minima, before bringing in more model-dependent information. For instance, as we will show later on, some constraints involving $\tan \beta$ and usually quoted in a model-dependent context, do have fully model-independent counterparts in the MSSM, which are even more stringent!

Getting fairly reasonable approximations of the effective potential is not always an easy task, for many reasons. Many papers have been devoted to these aspects in the MSSM (see for instance [5, 6]), however often with the main concern of reproducing a more or less quantitative pattern of the mass spectrum, at the expense of making some rough assumptions about what the radiative EWSB conditions actually are, and about the scale at which they are realized. This is of course fully justified, but only as a first approximation, as recognized by some authors [7]. The aim of the present paper is not 
to provide yet another tentative mass spectrum in the context of some approximations, but rather to fit into a more theoretical study of the validity of some of the underlying assumptions. Let us first list, for further reference, the features that should be kept in mind in this context:

i) The true effective potential $V_{\text {eff }}$ should be renormalization scale invariant. The renormalization group (RG) improved effective potential $\bar{V}_{\text {eff }}$ can miss large logarithms when there are more than one (mass) scale in the theory, as is the case in the MSSM. If not properly treated, the scale at which leading logs are actually resummed becomes fuzzy and with it the approximate scale invariance of $\bar{V}_{\text {eff }}$. ii) The only gauge parameter independent and physically relevant values of $V_{\text {eff }}$ are those at stationary points. iii) The "effective potential" computed from the 1PI Green's functions at vanishing external momenta, can suffer from unwanted concavities ( the true effective potential being always convex), precisely in the region where spontaneous symmetry breaking occurs. iv) A local minimum is characterized by both $1^{\text {st }}$ and $2^{\text {nd }}$ order derivatives with respect to all scalar fields in the theory. v) Non logarithmic contributions to $V_{\text {eff }}$ at a given loop order can alter the qualitative picture of the "tree-level improved" effective potential.

Another [perhaps less stressed] feature in connection with iv), is that what is usually called "the condition of electroweak symmetry breaking" and used as such in the literature, has to do, strictly speaking, only with the requirement of a stationary point of the effective potential in the Higgs directions. This obviously calls for an immediate explanation. In this paper we will show, in connection with iv) and $v$ ), that while the stationarity condition is sufficient, as far as the tree-level and "tree-level improved" effective potential are concerned, due to a non-trivial connection between $1^{\text {st }}$ and $2^{\text {nd }}$ order derivatives, it is no more the case when finite (non-logarithmic) one-loop effects are taken into account. Naively, one would argue that such effects cannot change much of the qualitative pattern of the tree-level minima. The fact is that, even if the one-loop effective potential is expected to differ, point-to-point, only perturbatively from its tree-level value, it is still possible that its shape be locally modified in such a way that new local minima (or at least stationary points) appear. This fact will bring in new supplementary conditions to the usual EWSB ones, determined analytically in a general context. These conditions will lead in turn to model-independent and calculable exclusion domains of $\tan \beta$, to compare with some existing model-dependent ones. Furthermore, one will have to assess the necessity and sufficiency for the new conditions. For this we will have to revisit briefly the question of charge breaking in the Higgs sector.

Another relevant aspect which we will consider, has to do with the gauge-fixing de- 
pendence of the derived conditions. We will give a simple argument in favour of the gauge-fixing independence, in the sense that the corresponding equations (and inequalities) remain satisfied even if there is some gauge-fixing dependence on either side of these equations, that is they are gauge-fixing "covariant".

The rest of the paper will be organized as follows. In section 2 we recall the main features of the effective potential in the MSSM and present some of the assumptions we make throughout the paper and conventions we use. Section 3 describes what we call the "tree-level logic" for the EWSB conditions. In section 4 we give an expression for Str $M^{4}$ in the Higgs sector, derive the new EWSB conditions at one-loop order in a fully analytic form, and show why the "tree-level logic" does not generally hold. We then derive the model-independent theoretical bounds on $\tan \beta$. The contents of this section represent the main results of the paper. In section 5 we discuss the meaning of our constraints and how they compare to the existing ones. The question of necessity and sufficiency of our conditions is addressed in section 6 . Section 7 is devoted to the question of gauge-fixing dependence. A first numerical illustration is given in section 8, in the context of SUGRAGUT scenarios. The conclusions are given in section 9, and some technical material and proofs relegated to the appendix. For a quick summary of the issues presented in this paper the reader is referred to [8]. This is part of a thorough analytic study of the complete one-loop effective potential in the MSSM, where symbolic computation is heavily used [9].

In this paper the word "stationarity" will refer to the vanishing of the $1^{\text {st }}$ order derivatives of the effective potential, and the word "stability" to the requirement that the eigenvalues of the $2^{\text {nd }}$ order derivatives should be positive. 


\section{Approximations and notations}

Our starting point will be the full fledged MSSM with a priori no model dependent assumptions whatsoever. The aim of the study is to assess the power of the only assumption of minimal supersymmetry in constraining the various free parameters, once electroweak symmetry breaking is required. It is nonetheless unavoidable, for practical purposes, to take up a definite point of view, as far as some approximations, usually made in a modeldependent context, are concerned. For instance, regarding point i) of the previous section, it has been emphasized in the literature that the mere replacement of the free parameters by their runnings in the tree-level effective potential, is actually a poor approximation [6]. A prominent drawback of this approximation is the rather high sensitivity of the "improved" effective potential to the renormalization scale. It was then observed that the same procedure applied to the one-loop effective potential leads to a better approximation, in the sense that the results are numerically much more stable against the change of renormalization scale. 6, 10.

One should, nevertheless, keep in mind that these approximations cannot be put in a neat context of leading- ( sub-leading-, etc...) log approximations anyway, as long as the case of many different mass and field scales is not properly handled [11]. For instance, there are still regions where the vev's still suffer a pronounced scale dependence, even to one-loop order [12]. In a sense, this should not come as a surprise since not all the logs can be properly resummed this way. For this reason, and since our aim is to study the effects of the non-logarithmic one-loop contributions, we will be contented throughout with the tree-level improved effective potential approximation.

The 1-loop effective potential has the well-known form [13]

$$
V=V_{\text {tree }}+\frac{\hbar}{64 \pi^{2}} \operatorname{Str}\left[M^{4}\left(\log \frac{M^{2}}{\mu_{R}^{2}}-3 / 2\right)\right]
$$

in the $\overline{M S}$ scheme. Here $\mu_{R}$ denotes the renormalization scale, $M^{2}$ the field dependent squared mass matrix of the scalar fields, and $\operatorname{Str}[\ldots] \equiv \sum_{\text {spin }}(-1)^{2 s}(2 s+1)(\ldots)_{s}$, where the sum runs over gauge boson, fermion and scalar contributions. $V_{\text {tree }}$ is the tree-level MSSM potential [14. As stated above, we will assume that the logarithms in Eq.(2.1) are reabsorbed in the running of all the parameters in $\bar{V}_{\text {tree }}\left(\mu_{R}^{2}\right)$. This amounts to the rough assumption of neglecting altogether all field and mass scale differences in the logs. The effective potential takes then the form

$$
V=\bar{V}_{\text {tree }}\left(\mu_{R}^{2}\right)+\frac{\hbar}{64 \pi^{2}}(-3 / 2) S t r M^{4}
$$

Here $\bar{V}_{\text {tree }}\left(\mu_{R}^{2}\right)$ is obtained from $V_{\text {tree }}$ by replacing all the tree-level quantities by their running counterparts. 
In our convention, the superpotential reads

$$
W=\sum_{i, j=g e n} Y_{i j}^{u} \tilde{u}_{R}^{i} H_{2} \cdot \tilde{Q}^{j}+Y_{i j}^{d} \tilde{d}_{R}^{i} H_{1} \cdot \tilde{Q}^{j}+Y_{i j}^{l} \tilde{l}_{R}^{i} H_{2} \cdot \tilde{L}^{j}+\mu H_{2} \cdot H_{1}
$$

where $\tilde{u}_{R}, \tilde{d}_{R}, \tilde{Q}$ (resp. $\tilde{l}_{R}, \tilde{L}$ ) denote squark (resp. slepton) right and left-handed scalar fields, and $H_{1}, H_{2}$ the Higgs scalar fields. The product between $S U(2)_{L}$ doublets reads $H . Q \equiv \epsilon_{i j} H^{i} Q^{j}$ where $i, j$ are $S U(2)_{L}$ indices.

The tree level potential $V_{\text {tree }}$ is the sum of the so-called F- and D-terms, where F-terms come from the superpotential through derivatives with respect to all scalar fields $\phi_{a}$

$$
V_{F}=\sum_{a}\left|W^{a}\right|^{2} \quad, \quad W^{a}=\frac{\partial W}{\partial \phi_{a}}
$$

and D-terms corresponding to respectively $U(1)_{Y}, S U(2)_{L}$, and $S U(3)_{C}$ gauge symmetries are given by

$$
V_{D}=D_{1} D_{1}+D_{2} D_{2}+D_{3} D_{3}
$$

with

$$
\begin{aligned}
D_{1}= & g_{1}^{2}\left[\sum_{i=g e n}\left(\frac{1}{6} \tilde{Q}_{i}^{\dagger} \tilde{Q}_{i}-\frac{1}{2} \tilde{L}_{i}^{\dagger} \tilde{L}_{i}-\frac{2}{3} \tilde{u}_{R_{i}}^{\dagger} \tilde{u}_{R_{i}}+\frac{1}{3} \tilde{d}_{R_{i}}^{\dagger} \tilde{d}_{R_{i}}+\tilde{l}_{R_{i}}^{\dagger} \tilde{l}_{R_{i}}\right)\right. \\
& \left.+\frac{1}{2} H_{2}^{\dagger} H_{2}-\frac{1}{2} H_{1}^{\dagger} H_{1}\right] \\
D_{2}= & g_{2}^{2}\left[\sum_{i=g e n}\left(\tilde{Q}_{i}^{\dagger} \frac{\vec{\sigma}}{2} \tilde{Q}_{i}+\tilde{L}_{i}^{\dagger} \frac{\vec{\sigma}}{2} \tilde{L}_{i}\right)+H_{2}^{\dagger} \frac{\vec{\sigma}}{2} H_{2}+H_{1}^{\dagger} \frac{\vec{\sigma}}{2} H_{1}\right] \\
D_{3}= & g_{3}^{2} \sum_{i=\text { gen }}\left[\tilde{Q}_{i}^{\dagger} \frac{\vec{\lambda}}{2} \tilde{Q}_{i}-\tilde{u}_{R_{i}}^{\dagger} \frac{\lambda^{*}}{2} \tilde{u}_{R_{i}}-\tilde{d}_{R_{i}}^{\dagger} \frac{\vec{\lambda}^{*}}{2} \tilde{d}_{R_{i}}\right]
\end{aligned}
$$

Here $g_{1}, g_{2}, g_{3}$ denote the three gauge couplings, $\left(\sigma_{k}\right)_{k=1, . ., 3}$ and $\left(\lambda_{k}\right)_{k=1, . ., 8}$ the Pauli and Gell-Mann matrices.

To calculate $\operatorname{Str} M^{4}$, we must also specify our conventions for the supersymmetric couplings of the scalar-fermion sector. Following the conventions of [15], with two-component spinors, this part of the Lagrangian contains on one hand a purely chiral sector

$$
\mathcal{L}_{\text {chir. }}=-\frac{1}{2} \sum_{a, b} W^{a b} \psi_{a} \psi_{b}+h . c \quad, \quad W^{a b}=\frac{\partial^{2} W}{\partial \phi_{a} \partial \phi_{b}}
$$

where $\psi_{a}$ is the supersymmetric fermionic partner of $\phi_{a}$, and on the other hand a mixed chiral-vector sector, which yields in component form

$$
\mathcal{L}_{\text {mix }}=i \sqrt{2} \sum_{A, a}\left(D_{A}\right)^{a} \psi_{a} \lambda_{A}+h . c \quad, \quad\left(D_{A}\right)^{a}=\frac{\partial D_{A}}{\partial \phi_{a}}
$$


The index A denotes the gauge group with which the gaugino $\lambda_{A}$ is associated.

The soft scalar supersymmetry breaking terms take the form

$$
\begin{aligned}
V \text { soft }= & m_{H_{2}}^{2} H_{2}^{\dagger} H_{2}+m_{H_{1}}^{2} H_{1}^{\dagger} H_{1}-B \mu\left(H_{1} \cdot H_{2}+h . c\right) \\
& +\sum_{i=g e n} m_{\tilde{Q}, i}^{2} \tilde{Q}_{i}^{\dagger} \tilde{Q}_{i}+m_{\tilde{L}, i}^{2} \tilde{L}_{i}^{\dagger} \tilde{L}_{i}+m_{\tilde{u}, i}^{2}\left|\tilde{u}_{R_{i}}\right|^{2}+m_{\tilde{d}, i}^{2}\left|\tilde{d}_{R_{i}}\right|^{2}+m_{\tilde{l}, i}^{2}\left|\tilde{l}_{R_{i}}\right|^{2} \\
& +\sum_{i, j=g e n}\left(A_{i j}^{u} Y_{i j}^{u} \tilde{u}_{R_{i}} H_{2} \cdot \tilde{Q}_{j}+A_{i j}^{d} Y_{i j}^{d} \tilde{d}_{R_{i}} H_{1} \cdot \tilde{Q}_{j}+A_{i j}^{l} Y_{i j}^{l} \tilde{l}_{R_{i}} H_{2} \cdot \tilde{L}_{j}+h . c\right)(2.1
\end{aligned}
$$

Finally, the gaugino soft mass term reads

$$
\mathcal{L}_{\text {mass }}^{\text {gaugino }}=-\frac{1}{2} \sum_{i=1}^{3} M_{i} \lambda_{i} \lambda_{i}+h . c
$$

We are interested here in the Higgs directions in which case $V_{\text {tree }}$ takes the form,

$$
\begin{aligned}
V_{\text {tree }}= & m_{1}^{2} H_{1}^{\dagger} H_{1}+m_{2}^{2} H_{2}^{\dagger} H_{2}+m_{3}^{2}\left(H_{1} \cdot H_{2}+\text { h.c. }\right) \\
& +\frac{g^{2}}{8}\left(H_{1}^{\dagger} H_{1}-H_{2}^{\dagger} H_{2}\right)^{2}+\frac{g_{2}^{2}}{2}\left(H_{1}^{\dagger} H_{2}\right)\left(H_{2}^{\dagger} H_{1}\right)
\end{aligned}
$$

where $g^{2} \equiv g_{1}^{2}+g_{2}^{2}, m_{1}^{2}=m_{H_{1}}^{2}+\mu^{2}, m_{2}^{2}=m_{H_{2}}^{2}+\mu^{2}, m_{3}^{2}=-B \mu$, and

$$
H_{1}=\left(\begin{array}{c}
H_{1}^{0} \\
H_{1}^{-}
\end{array}\right) \quad H_{2}=\left(\begin{array}{c}
H_{2}^{+} \\
H_{2}^{0}
\end{array}\right)
$$




\section{The "tree-level logic"}

In this section we will show, starting from the potential, Eq.(2.13), that

the requirement of boundedness from below of the potential, implies that any gauge non-invariant stationary point in the Higgs directions is necessarily a local minimum, and that the existence of such a point guarantees the instability of the gauge symmetric "vacuum".

This interesting property is common knowledge, but, as far as we know, is seldom stated clearly, let alone shown explicitly. We do this here for several reasons: First, to stress the fact that this property is not self-evident, but rather a consequence of the special form of Eq.(2.13), i.e. of (softly-broken) supersymmetry; secondly, because it underlies the reason for which the usual EWSB conditions

$$
\frac{1}{2} M_{Z}^{2}=\frac{\bar{m}_{1}^{2}-\bar{m}_{2}^{2} \tan ^{2} \beta}{\tan ^{2} \beta-1} \quad, \quad \sin 2 \beta=\frac{-2 \bar{m}_{3}^{2}}{\bar{m}_{1}^{2}+\bar{m}_{2}^{2}}
$$

used in the literature as minimization conditions, but which are strictly speaking only stationarity conditions, happen to be safe, i.e. necessary and sufficient, as far as the effective potential has the form of eq.(2.13). We refer to this situation as the "tree-level logic", even though it obviously holds as well in the tree-level-RG improved case; thirdly, to set the stage for the next section where the one-loop case will be worked out in detail and shown to infringe the tree-level logic.

Since we are mainly interested here in whether a stationary point in the electroweak direction is a local minimum or not, one could argue that it is enough to choose from the start the (electrically) neutral directions

$$
<H_{1}>=\frac{1}{\sqrt{2}}\left(\begin{array}{c}
v_{1} \\
0
\end{array}\right) \quad<H_{2}>=\frac{1}{\sqrt{2}}\left(\begin{array}{c}
0 \\
v_{2}
\end{array}\right)
$$

as the relevant dynamical variables. However, this needs further justification. The doublets $H_{1}, H_{2}$ depend each on four real-valued fields so that, as stated in the introduction (point iv)), one should determine $1^{\text {st }}$ and $2^{\text {nd }}$ order derivatives of $V_{\text {tree }}$ with respect to the eight real fields, and then take $H_{1}=<H_{1}>, H_{2}=<H_{2}>$ 3. Nonetheless, it turns out that all $1^{\text {st }}$ order derivatives other than those with respect to the two neutral real

\footnotetext{
${ }^{3}$ Note that one can use the $S U(2)$ gauge freedom to take away three of the eight fields, but the question remains essentially the same.
} 
components, vanish trivially at $H_{1}=<H_{1}>, H_{2}=<H_{2}>$. The two usual stationarity conditions are then indeed the only ones to consider and can be readily obtained from Eq. (2.13) as

$$
\begin{aligned}
& m_{1}^{2} v_{1}+m_{3}^{2} v_{2}+\frac{g^{2}}{8} v_{1}\left(v_{1}^{2}-v_{2}^{2}\right)=0 \\
& m_{2}^{2} v_{2}+m_{3}^{2} v_{1}+\frac{g^{2}}{8} v_{2}\left(v_{2}^{2}-v_{1}^{2}\right)=0
\end{aligned}
$$

On the contrary, the $2^{\text {nd }}$ order derivatives of the potential yield à priori non trivial information in the eight field directions. The stability conditions are obtained using the invariants method (see appendix B). Substituting for $m_{1}$ and $m_{2}$ using Eqs. (3.3, 3.4) one finds five stability conditions,

$$
\begin{aligned}
& -\left(v_{1}^{2}+v_{2}^{2}\right) \frac{m_{3}^{2}}{v_{1} v_{2}} \geq 0 \\
& -\left(v_{1}^{2}-v_{2}^{2}\right)^{2} \frac{g^{2} m_{3}^{2}}{4 v_{1} v_{2}} \geq 0 \\
& \frac{\left(v_{1}^{2}+v_{2}^{2}\right)\left(-4 m_{3}^{2}+g^{2} v_{1} v_{2}\right)}{4 v_{1} v_{2}} \geq 0 \\
& \frac{\left(-4 m_{3}^{2}+g_{2}^{2} v_{1} v_{2}\right)}{4 v_{1} v_{2}} \geq 0 \quad \text { (twice) }
\end{aligned}
$$

plus three zeros corresponding to the three goldstone degrees of freedom. Eqs.(3.3 - 3.8) are the necessary and sufficient conditions for the existence of a local minimum in the electroweak sectort. We will show now that Eqs.(3.3, 3.4) imply automatically Eqs.(3.5) 3.8).

Let us first solve for $\tan \beta\left(\equiv \frac{v_{2}}{v_{1}}\right)$ in Eqs.(3.3, 3.4). One easily finds ,

$$
\tan \beta=\frac{-m_{1}^{2}-m_{2}^{2} \pm \sqrt{\left(m_{1}^{2}+m_{2}^{2}\right)^{2}-4 m_{3}^{4}}}{2 m_{3}^{2}}
$$

It is worthwhile noting at this level that the existence of real solutions in Eq.(3.9) is guaranteed by the requirement of boundedness from below of $V_{\text {tree }}$. The conditions of boundedness from below involve necessarily the soft supersymmetry breaking parameters and reside entirely in the would-be "flat directions" of the potential. The obvious reason

\footnotetext{
${ }^{4}$ It should be clear that they are not sufficient to ensure a global minimum, the latter necessitating the comparison of various local minima, including those outside the Higgs directions.
} 
for this is that in exact supersymmetry the potential is positive semi-definite so that only explicit soft breaking terms can make it unbounded from below. Furthermore, the directions along which the potential can turn down negative first, when the soft terms are switched on, are those for which the supersymmetric potential is the closest to zero at arbitrarily large fields. This happens generically In the case of Eq.(2.13), the flat directions are D-flat and correspond to

$$
\left|H_{1}\right|=\left|H_{2}\right| \text { and } \quad H_{1}^{\dagger} H_{2}=0
$$

A representative direction is, (modulo a gauge transformation),

$$
H_{1}=\left(\begin{array}{c}
H \\
0
\end{array}\right) \quad H_{2}=\left(\begin{array}{c}
0 \\
\pm H
\end{array}\right)
$$

where $\mathrm{H}$ denotes any complex valued field. $V_{\text {tree }}$ is quadratic in $\mathrm{H}$ along such directions, and the boundedness from below conditions (BFB) read,

$$
m_{1}^{2}+m_{2}^{2} \pm 2 m_{3}^{2} \geq 0
$$

With the above conditions, $\tan \beta$ in Eq.(3.9) is real valued.

More importantly, further inspection of Eq.(3.9), in conjunction with Eq.(3.12), tells us that $\tan \beta$ and $m_{3}^{2}$ have opposite signs, that is

$$
\frac{m_{3}^{2}}{v_{1} v_{2}} \leq 0
$$

A quick look at the stability conditions Eqs.(3.5 - 3.8) shows then that they are all automatically satisfied.

On the other hand, substituting for $m_{1}$ and $m_{2}$, one gets from $\operatorname{Eqs}(3.3,3.4)$,

$$
m_{1}^{2} m_{2}^{2}-m_{3}^{4}=-\frac{1}{64} g^{2}\left(v_{1}^{2}-v_{2}^{2}\right)^{2}\left(g^{2}-8 m_{3}^{2} /\left(v_{1} v_{2}\right)\right)
$$

so that $\mathrm{Eq}(3.13)$ implies readily

$$
m_{1}^{2} m_{2}^{2}-m_{3}^{4}<0
$$

i.e., the potential is automatically unstable at zero vev's.

Up to this level, we have used only partially the conditions assuring the existence of a stationary point away from the origin. In particular, the existence of $\tan \beta$ solutions,

\footnotetext{
${ }^{5}$ barring the occurrence of runaway vacua,
} 
Eq.(3.9), does not guarantee the reality of the vev's, i.e. $v_{1}^{2}, v_{2}^{2}>0$. To see this, let us extract from Eqs.( 3.3, 3.4) the equation complementary to Eq.(3.9), namely,

$$
u \equiv v_{1} v_{2}=\frac{8 t}{g^{2}\left(1-t^{4}\right)}\left(t^{2} m_{2}^{2}-m_{1}^{2}\right)
$$

where $t \equiv \tan \beta$. It is clear from the above equation that requiring $t$ and $u$ to have the same sign, i.e. $v_{1}^{2}, v_{2}^{2}>0$, will lead to extra relations among the $m_{i}{ }^{2}$ 's. The discussion can be carried out in terms of the sign of $m_{2}^{2}$ (assuming throughout $m_{1}^{2}>0$, as is generically the case in susy-GUT scenarios). If $m_{2}^{2}<0$, which is often the case at the electroweak scale 9 , then one has from Eq.(3.16) and ut $>0$ that $|\tan \beta|<1$ is excluded, and otherwise no extra conditions on the $m_{i}{ }^{2}$ 's. If however $m_{2}^{2}>0$, then one can show, given Eqs.3.9, 3.16) and the BFB Eq.(3.12), that the sufficient and necessary conditions for $u t>0$ are simply

$$
m_{1}^{2} m_{2}^{2}-m_{3}^{4}<0 \text { and } m_{1}^{2}>m_{2}^{2}\left(\text { resp. } m_{1}^{2}<m_{2}^{2}\right) \text { for }|t|>1 \quad(\text { resp. }|t|<1)
$$

The first of these two conditions is, however, already a consequence of Eq.(3.9) as we have shown above.

To summarize, we have shown in this section that the requirement of boundedness from below Eq.(3.12) and the conditions of existence of a stationary point at non-vanishing field values Eqs(3.3, 3.4), imply by themselves that this point is a local minimum Eqs.(3.5 3.8), and that the gauge symmetric point, $v_{1}=v_{2}=0$ is energetically unstable Eq.(3.14). On the other hand, the discussion following Eq.(3.16) is to remind us that even at treelevel, the model-independent EWSB conditions imply some constraints on the allowed values of $\tan \beta$, depending on the relative magnitude and/or signs of $m_{1}^{2}, m_{2}^{2}$. Of course, if one implements the information coming from say a SUGRA-GUT scenario together with universality assumptions then the above constraints become stricter as $m_{1}^{2}, m_{2}^{2}$ run down from their initial values in a well-defined way. In particular, theoretical upper bounds are known to appear ([16]). The main point of the next section is to show that similar constraints obtain when one considers the one-loop EWSB constraints, irrespective of model-dependent assumptions.

Finally a couple of remarks are in order.

- First, the fact that the second order derivatives are automatically positive when the first order ones are vanishing can of course be also seen from the tree-level MSSM relations among the Higgs masses. Indeed the well-known relations

\footnotetext{
${ }^{6}$ Remember that $m_{2}^{2}<0$ is only sufficient to ensure an unstable gauge-invariant vacuum, the necessary (and sufficient) condition being Eq. (3.15)
} 


$$
\begin{gathered}
m_{h^{0}, H^{0}}^{2}=\frac{1}{2}\left(m_{Z}^{2}+m_{A^{0}}^{2} \mp \sqrt{\left(m_{Z}^{2}+m_{A^{0}}^{2}\right)^{2}-4 m_{Z}^{2} m_{A^{0}}^{2} \cos ^{2} 2 \beta}\right) \\
m_{H^{ \pm}}^{2}=m_{A^{0}}^{2}+m_{W}^{2}
\end{gathered}
$$

are such that all squared Higgs masses are automatically positive once $m_{A}^{2} \geq 0$. Furthermore, since

$m_{A}^{2}=-\left(v_{1}^{2}+v_{2}^{2}\right) \frac{m_{3}^{2}}{v_{1} v_{2}}$, the positivity of $m_{A}^{2}$ is itself a consequence of Eq.(3.13), which in turn is implied by the vanishing of the first order derivatives. It thus follows that the positivity of all the squared Higgs masses is at tree-level a consequence of just the stationarity conditions.

- sign of $\tan \beta$ : For later discussion, let us also recall the reason why $\tan \beta$ can always be chosen positive at tree-level. As already stated, Eq.(3.9) implies by itself that the two $\tan \beta$ solutions have the same sign, opposite to that of $m_{3}^{2}$. The latter can always be chosen negative in Eq.(2.13) by properly choosing the relative phase of $H_{1}$ and $H_{2}$. One can thus replace from the start $m_{3}^{2}$ by $-\left|m_{3}^{2}\right|$ in Eq. (2.13) and choose all the way long $\tan \beta>0$. [The choice of positive $\tan \beta$ will still be possible beyond tree-level, however in a more tricky way. Indeed, as we will see in the next section, the signs of $\tan \beta$ and the one-loop corrected $m_{3}^{2}$ are no more directly connected through the stationarity conditions].

- The case $\tan \beta=1$ : Whatever the theoretical $\tan \beta$ exclusion domains turn out to be, we would like to stress that the value $\tan \beta=1$ should be excluded. As can be easily seen from Eqs.(3.3, 3.4), when $v_{1}=v_{2}($ and $\neq 0)$ one has necessarily $m_{1}^{2}+m_{2}^{2}+2 m_{3}^{2}=0$. This implies that the tree-level potential Eq.(2.13) is vanishing everywhere in the neutral direction Eq.(3.2) so that there is no dynamically preferred electroweak minimum. As we will see in the next section, although the degeneracy is lifted by the one-loop corrections, flatness remains in the $v_{1}=v_{2}$ direction in such a way that the stationary point at $\tan \beta=1$ is not a minimum.

\section{$4 \quad \operatorname{Str} M^{4}$ and the 1-loop EWSB conditions}

The natural question to ask, given the results of the previous section, is whether the non-trivial connection between stationarity and local stability just demonstrated, can hold beyond the tree-level. For one thing, it is obvious that it does in the tree-levelRGE improved approximation, where the potential retains its tree-level field dependence, provided that multi-scale subtleties be momentarily evaded by assuming roughly one 
energy scale, and making the substitutions $m_{i}^{2} \rightarrow \bar{m}_{i}^{2}\left(\mu_{R}^{2}\right), g_{i} \rightarrow \bar{g}_{i}\left(\mu_{R}^{2}\right)$. [The running of the Higgs fields being quite small is usually disregarded. In any case the implicit $\mu_{R}^{2}$ dependence on the fields is ignored within the approximation, so that one can keep theses fields fixed for the purpose of minimization.]

Potentially new information would come from the study of the non-logarithmic oneloop correction to the effective potential, which we turn to now. Here we stick as before to the $H_{1}, H_{2}$ directions, and keep as much as possible the discussion at a model-independent level.

Putting together the contributions of the full-fledged MSSM, we find the following form for the one-loop effective potential in the Landau gauge, in the Higgs directions 6 ,

$$
\begin{aligned}
V_{\text {tree }}+\kappa S \operatorname{Str} M^{4}= & X_{m_{1}}^{2} H_{1}^{\dagger} H_{1}+X_{m_{2}}^{2} H_{2}^{\dagger} H_{2}+X_{m_{3}}^{2}\left(H_{1} \cdot H_{2}+\text { h.c. }\right) \\
& +X\left(H_{1}^{\dagger} H_{1}-H_{2}^{\dagger} H_{2}\right)^{2}+\tilde{\beta}\left|H_{1}^{\dagger} H_{2}\right|^{2}+\tilde{\alpha}\left(\left(H_{1}^{\dagger} H_{1}\right)^{2}-\left(H_{2}^{\dagger} H_{2}\right)^{2}\right)+\Omega_{0}
\end{aligned}
$$

where

$$
\begin{aligned}
& \tilde{\alpha}=\frac{3}{2} \kappa g^{2}\left(Y_{t}^{2}-Y_{b}^{2}\right) \\
& \tilde{\beta}=\frac{g_{2}^{2}}{2}+\kappa g_{2}^{2}\left(g_{1}^{2}+5 g_{2}^{2}-6\left(Y_{t}^{2}+Y_{b}^{2}\right)\right) \\
& X=\frac{g^{2}}{8}+\kappa\left(g_{1}^{2} g_{2}^{2}+\frac{23 g_{1}^{4}+5 g_{2}^{4}}{4}-\frac{3}{2} g^{2}\left(Y_{t}^{2}+Y_{b}^{2}\right)\right) \\
& X_{m_{1}}^{2}=m_{H_{d}}^{2}+\mu^{2}+\kappa\left[-4 g_{1}^{2} M_{1}^{2}+3 g_{2}^{2}\left(m_{H_{d}}^{2}-3 \mu^{2}-4 M_{2}^{2}\right)\right. \\
& +12\left(Y_{b}^{2}\left(A_{b}^{2}+m_{\tilde{b_{R}}}^{2}+m_{\tilde{T}}^{2}\right)+\mu^{2} Y_{t}^{2}\right) \\
& +g_{1}^{2}\left(3 m_{H_{d}}^{2}-2 m_{H_{u}}^{2}-3 \mu^{2}\right. \\
& \left.\left.-\sum_{i=\text { generation }} 2\left(m_{\tilde{d}_{R}, i}^{2}+m_{\tilde{l}_{R}, i}^{2}+m_{\tilde{Q}, i}^{2}-m_{\tilde{L}, i}^{2}-2 m_{\tilde{u}_{R}, i}^{2}\right)\right)\right] \\
& X_{m_{2}}^{2}=m_{H_{u}}^{2}+\mu^{2}+\kappa\left[-4 g_{1}^{2} M_{1}^{2}+3 g_{2}^{2}\left(m_{H_{u}}^{2}-3 \mu^{2}-4 M_{2}^{2}\right)\right. \\
& +12\left(Y_{t}^{2}\left(A_{t}^{2}+m_{\tilde{t_{R}}}^{2}+m_{\tilde{T}}^{2}\right)+\mu^{2} Y_{b}^{2}\right) \\
& +g_{1}^{2}\left(3 m_{H_{u}}^{2}-2 m_{H_{d}}^{2}-3 \mu^{2}\right. \\
& \left.\left.+\sum_{i=\text { generation }} 2\left(m_{\tilde{d}_{R}, i}^{2}+m_{\tilde{l}_{R}, i}^{2}+m_{\tilde{Q}, i}^{2}-m_{\tilde{L}, i}^{2}-2 m_{\tilde{u}_{R}, i}^{2}\right)\right)\right]
\end{aligned}
$$

\footnotetext{
${ }^{7}$ the full analytic form of $\operatorname{Str} M^{4}$ in all scalar field directions will be given elsewhere [9]
} 


$$
\begin{aligned}
X_{m_{3}}^{2} & =-B \mu+\kappa \mu\left[g_{1}^{2}\left(B+4 M_{1}\right)+3 g_{2}^{2}\left(B+4 M_{2}\right)\right. \\
\kappa & =\left(-\frac{3}{2}\right) \frac{\hbar}{64 \pi^{2}} \\
g^{2} & \equiv g_{1}^{2}+g_{2}^{2}
\end{aligned}
$$

The above expressions are exact, apart from the fact that we kept only the top/bottom Yukawa couplings $Y_{t}$ and $Y_{b}$ for simplicity, the generalization to the other Yukawa couplings being easy to implement, and assumed diagonal and real Yukawa matrices. The $X_{m_{i}}^{2}$ 's are functions of the various soft susy breaking masses and couplings, associated with all squark-doublet-masses, $m_{\tilde{Q}, i}, m_{\tilde{T}}$, and singlet- masses, $m_{\tilde{u}_{R}, i}, m_{\tilde{d}_{R}, i}, m_{\tilde{b}_{R}}, m_{\tilde{t}_{R}}$, all slepton-doublet-masses , $m_{\tilde{L}, i}$ and singlets masses $m_{\tilde{l}_{R}, i}$ (no right-handed $\tilde{\nu}$ ), gaugino soft masses $M_{1}, M_{2}$ (no gluino contributions at this level in the Higgs directions), Higgs soft masses $m_{H_{1}}^{2}, m_{H_{2}}^{2}$ and the supersymmetric $\mu$-term related to $m_{1}^{2}, m_{2}^{2}, m_{2}^{3}$ as defined in section 2, and the soft trilinear couplings $A_{t}, A_{b}$. Note also that $\Omega_{0}$ in Eq.(4.1) is a field independent additive constant depending exclusively on soft susy breaking terms. It contributes to the cosmological constant and will be discarded throughout $\mathrm{O}$. We stress that no model-dependent assumptions were used to establish the above expressions. The results which will follow from them will thus be applicable either in the context of SUGRA-GUT scenarios, where eventually universality may be assumed [2], or in a gauge-mediated supersymmetry breaking context [3], or for that matter in any fully model-independent analysis.

Boundedness from below. The would-be flat directions remain (as expected) the same as at tree-level, i.e.

$$
\left|H_{1}\right|=\left|H_{2}\right| \text { and } H_{1}^{\dagger} H_{2}=0
$$

and give the following one-loop condition for a bounded-from-below potential,

$$
X_{m_{1}}^{2}+X_{m_{2}}^{2} \pm 2 X_{m_{3}}^{2} \geq 0
$$

Stationarity conditions. From Eq.(4.1) we find the stationarity conditions with respect to the 8 real components in $H_{1}, H_{2}$ at $H_{1}=<H_{1}>, H_{2}=<H_{2}>$. As in the previous section, they boil down to two conditions,

$$
X_{m_{1}}^{2} v_{1}+X_{m_{3}}^{2} v_{2}+X v_{1}\left(v_{1}^{2}-v_{2}^{2}\right)+\tilde{\alpha} v_{1}^{3}=0 \quad, \quad X_{m_{2}}^{2} v_{2}+X_{m_{3}}^{2} v_{1}+X v_{2}\left(v_{2}^{2}-v_{1}^{2}\right)-\tilde{\alpha} v_{2}^{3}=0
$$

\footnotetext{
${ }^{8}$ see however 11] for relevant issues
} 
It is suitable for the subsequent discussion to express these equations in terms of $t \equiv \tan \beta$ and $u \equiv v_{1} v_{2}$ as follows,

$$
\begin{gathered}
X_{m_{3}}^{2}(\tilde{\alpha}-X) t^{4}+\left(\tilde{\alpha} X_{m_{1}}^{2}-X\left(X_{m_{1}}^{2}+X_{m_{2}}^{2}\right)\right) t^{3} \\
+\left(\tilde{\alpha} X_{m_{2}}^{2}+X\left(X_{m_{1}}^{2}+X_{m_{2}}^{2}\right)\right) t+X_{m_{3}}^{2}(\tilde{\alpha}+X)=0 \\
u=\frac{1}{\tilde{\alpha}\left(t^{2}-1\right)}\left(X_{m_{3}}^{2}\left(t^{2}+1\right)+\left(X_{m_{1}}^{2}+X_{m_{2}}^{2}\right) t\right)
\end{gathered}
$$

Let us pause here and make some important remarks for what will follow. The variable $\tilde{\alpha}$ defined in Eq.(4.2), which is thoroughly a one-loop contribution, will play a central role in the analysis leading to the new model-independent constraints on $\tan \beta$. The fact that it modifies, even though numerically slightly, the structure of the tree-level quartic terms in the effective potential will generically invalidate the tree-level logic of the connection between $1^{\text {st }}$ and $2^{\text {nd }}$ order derivatives. Furthermore, $\tilde{\alpha}$ is proportional to the difference between up and down Yukawa couplings. This will be at the origin of the $\tan \beta$ bounds involving $\frac{m_{t}}{m_{b}}$. Finally, $\tilde{\alpha}$ modifies drastically the $\tan \beta$ dependence of the stationarity conditions. Indeed, Eqs. 3.3, 3.4) lead to an equation quadratic in $\tan \beta$, while Eq. (4.13) is quartic, so that one should expect generically up to four possible real values for $\tan \beta$. As we will see, and already remarked in section 3, it would be erroneous then to discard from the start the negative valued ones.

Stability conditions. We now go a little further and determine from Eq.(4.1) the $2^{\text {nd }}$ order derivative matrix with respect to the eight real valued components of $H_{1}, H_{2}$, and extract from it (using the invariants method described appendix B), five stability conditions, plus, as expected, three zeros corresponding to the goldstone modes. We find, (after having eliminated $X_{m_{1}}^{2}, X_{m_{2}}^{2}$ through Eqs.(4.12)),

$$
\begin{gathered}
-\left(v_{1}^{2}+v_{2}^{2}\right) \frac{X_{m_{3}}^{2}}{v_{1} v_{2}} \geq 0 \\
2 \tilde{\alpha}\left(v_{1}^{2}-v_{2}^{2}\right)+\left(v_{1}^{2}+v_{2}^{2}\right)\left(2 X-\frac{X_{m_{3}}^{2}}{v_{1} v_{2}}\right) \geq 0 \\
-4 \tilde{\alpha}^{2} v_{1}^{2} v_{2}^{2}+2\left(v_{2}^{2}-v_{1}^{2}\right)\left[\left(v_{1}^{2}+v_{2}^{2}\right) \tilde{\alpha}-\left(v_{2}^{2}-v_{1}^{2}\right) X\right] \frac{X_{m_{3}}^{2}}{v_{1} v_{2}} \geq 0
\end{gathered}
$$

\footnotetext{
${ }^{9}$ recall that we consider here just the top/bottom doublet
} 


$$
\left(-\frac{X_{m_{3}}^{2}}{v_{1} v_{2}}+\tilde{\beta}\right)\left(v_{1}^{2}+v_{2}^{2}\right) \geq 0 \text { (twice) }
$$

Eq.(4.15) is clearly equivalent to

$$
\frac{X_{m_{3}}^{2}}{v_{1} v_{2}} \leq 0
$$

which is the straightforward one-loop generalization of Eq.(3.13). Relying on the fact that the inequalities $X \pm \tilde{\alpha} \geq 0$ and $\tilde{\beta} \geq 0$ are always perturbatively satisfied, one shows easily that Eqs.4.16, 4.18) hold as a consequence of Eq.(4.19).

We are then left with Eq.(4.17). It can be easily shown that this equation is equivalent to

$$
\tan ^{2} \beta \leq t_{-} \text {or } \tan ^{2} \beta \geq t_{+}
$$

where

$$
t_{ \pm}=\frac{\tilde{\alpha}^{2} \frac{v_{1} v_{2}}{X_{m_{3}}^{2}}-X \mp \sqrt{\left(X-\tilde{\alpha}^{2} \frac{v_{1} v_{2}}{X_{m_{3}}^{2}}\right)^{2}+\tilde{\alpha}^{2}-X^{2}}}{\tilde{\alpha}-X}
$$

Note that in writing the above equations we assumed that Eq.(4.19) is verified, so that $t_{ \pm}$ are both positive and satisfy $t_{+} \geq t_{-}$, since $X$ and $X-\tilde{\alpha}$ are both perturbatively positive.

To summarize, Eqs(4.19, 4.20) constitute new stability constraints, which are necessary for EWSB, and should à priori be superimposed on Eqs.(4.13,4.14).

The question we address now is whether these stability conditions are implicitly encoded in the stationarity equations (4.12), as was the case at the tree-level, or whether they are genuinely extra constraints. The answer here is technically much less trivial, for two reasons:

-the rather involved form of the bounds $t_{ \pm}$ -starting from the analytic solutions for Eq.(4.13), one can exhibit cases, compatible with boundedness from below Eq.4.11), where $\tan \beta$ and $X_{m_{3}}^{2}$ do have the same sign, contrary to what was going on at tree-level.

$\frac{X_{m_{3}}^{2}}{v_{1} v_{2}} \leq 0$ and $\tan \beta$ bounds. As far as Eq. (4.19) is concerned, the way out resides in Eq.(4.14) together with the requirement that $u$ and $t$ should have the same sign; we will show that Eq.(4.14) encodes enough information to imply new lower and upper modelindependent bounds on $\tan \beta$. [The automatic validity of Eq.(4.19) will persist only when $\left.1<|\tan \beta|<\frac{m_{t}}{m_{b}}\right]$. 
Let us give first a heuristic argument to illustrate how the interplay between Eqs.(4.14), (4.19) and (4.2) forbids $\tan \beta$ to be arbitrarily large or small. If the region $\tan \beta>>1$ were allowed, then Eq.(4.14) would behave like

$$
v_{1} v_{2} \sim \frac{X_{m_{3}}^{2}}{\tilde{\alpha}}
$$

in that region. Taking into account Eq.(4.19) one then must have

$$
\tilde{\alpha} \leq 0
$$

However, due to the form of $\tilde{\alpha}$, Eqs. (4.2, 4.8), and to the fact that $\tan \beta=\frac{Y_{b}}{Y_{t}} \frac{m_{t}}{m_{b}}$ one sees easily that $\tilde{\alpha} \leq 0$ and $\tan \beta$ arbitrarily large (i.e. $\frac{Y_{b}}{Y_{t}}>>1$ ) cannot be simultaneously satisfied 10 . We are thus lead to the conclusion that an arbitrarily large $\tan \beta$ would lead to a contradiction, i.e. there should be a theoretical upper bound on $\tan \beta$.

Similarly, in the region $|\tan \beta|<<1$ one has

$$
v_{1} v_{2} \sim-\frac{X_{m_{3}}^{2}}{\tilde{\alpha}}
$$

which, together with Eq.(4.19) implies that $\tilde{\alpha} \geq 0$, the latter inequality being in contradiction with $|\tan \beta|<<1$. Thus $\tan \beta$ cannot be arbitrarily small and a theoretical lower bound should exist. To determine the actual upper and lower bounds on $\tan \beta$ requires much more work. Here we only state the results, assuming without loss of generality, that $\tan \beta$ is positive (and $X_{m_{3}}^{2}<0$ ). The complete proof and further comments, including the correlation with the sign of $\tilde{\alpha}$, are deferred to appendix A. We find the following bounds:

$$
\begin{array}{ll}
\text { if } \tan \beta>1 & : \tan \beta_{-} \leq \tan \beta \leq \tan \beta_{+} \\
\text {where } & \tan \beta_{-}=\operatorname{Min}\left(T_{+}, \frac{m_{t}}{m_{b}}\right) \\
\text { and } & \tan \beta_{+}=\operatorname{Max}\left(T_{+}, \frac{m_{t}}{m_{b}}\right) \\
\text { if } \tan \beta<1 \quad: \quad T_{-} \leq \tan \beta<1
\end{array}
$$

\footnotetext{
${ }^{10}$ One should note that we rely here on the tree-level relations $m_{t}=\frac{Y_{t} v_{2}}{\sqrt{2}}, m_{b}=\frac{Y_{b} v_{1}}{\sqrt{2}}$, which, apart from leading log corrections in the running of $Y_{t}, Y_{b}, v_{i}$, might suffer from some small corrections. Nonetheless, the qualitative form of the constraint will not be altered
} 


$$
\text { where } T_{ \pm}=\frac{-X_{m_{1}}^{2}-X_{m_{2}}^{2} \mp \sqrt{\left(X_{m_{1}}^{2}+X_{m_{2}}^{2}\right)^{2}-4 X_{m_{3}}^{4}}}{2 X_{m_{3}}^{2}}
$$

the $X_{m_{i}}^{2}$ 's being given by Eqs. $(4.6$ - 4.8). It is interesting to note at this level the possibility of predicting the size (or even closing at will) the allowed window in the case $\tan \beta>1$. This depends on whether $\frac{m_{t}}{m_{b}}$ is greater or smaller than $T_{+}$, (the latter being controlled by the values of the free parameters of the MSSM). For instance in the parameter space region where

$$
X_{m_{1}}^{2}+X_{m_{2}}^{2} \simeq \frac{m_{t}}{m_{b}}\left|X_{m_{3}}^{2}\right|
$$

$\tan \beta$ is bound to lie very close to $\frac{m_{t}}{m_{b}}$ !

Finally, as we will also explicitly show in appendix A, the stability condition Eq.4.19) is not a consequence of the stationarity conditions, when $\tan \beta<1$ or $>\frac{m_{t}}{m_{b}}$ while it becomes so if $1<\tan \beta<\frac{m_{t}}{m_{b}}$. We find it rather noteworthy that the "tree-level logic" of section 2 , holds only in the region of $\tan \beta$ which is favoured by SUGRA-GUT scenarios, [16]!

the $t_{ \pm}$bounds. The $T_{ \pm}$are only part of the story. It is of course also necessary to take into account the stability conditions Eq.(4.20). When combining the bounds from Eqs.(4.25,4.26) with those from Eq.4.20) it will be important to understand the relative magnitudes of $\sqrt{t_{ \pm}}$as compared to $T_{ \pm}$and $\frac{m_{t}}{m_{b}}$, in order to tell when the new $t_{ \pm}$bounds become relevant. A complete answer to this would get too technical. Actually there is no generic trend for the relative magnitudes, so that firm conclusions can be drawn only numerically and will depend on the model assumptions. One can, however, show analytically that there is no obstruction for $\sqrt{t_{+}}$to lie within the interval $\left[\tan \beta_{-}, \tan \beta_{+}\right]$. This means that the tree-level connection between stationarity and stability is generally lost, even though it is partially preserved for Eq.(4.19), as was stated at the end of the previous paragraph. Hereafter we give, without proof, some of the salient properties of $t_{ \pm}$

Let us note first that the behaviour of $t_{ \pm}$in terms of $\zeta=\frac{\tilde{\alpha}^{2} v_{1} v_{2}}{X_{m_{3}}^{2}}$ is such that $0 \leq t_{-} \leq 1$ and $t_{+} \geq 1$, whatever the actual magnitude of $\zeta$ and the sign and magnitude of $\tilde{\alpha}$, provided that Eq.4.19) is verified, i.e. $\zeta \leq 0$. The proof of this is straightforward, and we will simply exemplify it in Fig. 1; thus a calculable region around $\tan \beta=1$ is always excluded by the stability constraint Eq.(4.20), the limiting value $\tan \beta=1$ being reached only when $\zeta=0$. On the other hand, $t_{ \pm}$depend on $t(\equiv \tan \beta)$ through $u\left(\equiv v_{1} v_{2}\right)$, while the bounds $T_{ \pm}$do not. In view of Eq.(4.14), one should treat with some care the two regimes $\tilde{\alpha}<<1$ 
and $t \sim 1$. In the regime $\tilde{\alpha}<<1$ and $t \neq 1, u$ is not singular, due to the fact that the numerator of Eq.(4.14) is $O(\tilde{\alpha})$ when Eq.(4.13) is taken into account. One finds, in the limit $\tilde{\alpha} \rightarrow 0$ four solutions, $-1,1, T_{+}, T_{-}$. Excluding $|t|=1$, the behaviour of $u$ is then given by

$$
\begin{gathered}
u \sim \frac{t}{X\left(1-t^{4}\right)}\left(t^{2} X_{m_{2}}^{2}-X_{m_{1}}^{2}\right)+O(\tilde{\alpha}) \\
\text { where } \quad t=T_{ \pm} .
\end{gathered}
$$

Note that this regime is relevant either when the one-loop correction is assumed to be small, or when $Y_{t} \sim Y_{b}$, i.e. $\tan \beta \sim \frac{m_{t}}{m_{b}}$. In the first case, one should also make the substitutions $X_{m_{i}}^{2} \rightarrow m_{i}^{2}, X \rightarrow \frac{g^{2}}{8}$, thus recovering Eq.(3.16).

The regime $t \simeq 1$ is somewhat more tricky. When $t \rightarrow 1$ one has simultaneously

$$
X_{m_{1}}^{2}+X_{m_{2}}^{2}+2 X_{m_{3}}^{2} \rightarrow 0
$$

as can be easily seen from Eqs.(4.12), and the one-loop effective potential comes very close to flat in the $v_{1} \sim v_{2}$ region. We thus parameterize this regime by the small number $\epsilon=X_{m_{1}}^{2}+X_{m_{2}}^{2}+2 X_{m_{3}}^{2}$. To understand the effect of non-vanishing $\tilde{\alpha}$ we consider the asymptotic region where $\epsilon<<\tilde{\alpha}$. When $\epsilon$ is vanishing, Eq.(4.13) has only one solution $t=1$. Barring the solution $t=-1$ without loss of generality, we find three positive solutions which are away from 1 by at least $O(\sqrt{\tilde{\alpha}})$ :

$$
\begin{aligned}
& t_{1}=1+\frac{\epsilon}{X_{m_{2}}^{2}-X_{m_{1}}^{2}}+O\left(\epsilon^{2}, \tilde{\alpha}\right) \\
& t_{2}=1+\frac{\epsilon}{2\left(X_{m_{1}}^{2}-X_{m_{2}}^{2}\right)}+\sqrt{\frac{\tilde{\alpha}\left(X_{m_{2}}^{2}-X_{m_{1}}^{2}\right)}{X\left(X_{m_{2}}^{2}+X_{m_{1}}^{2}\right)}}+O\left(\epsilon^{2}, \tilde{\alpha}\right) \\
& t_{3}=1+\frac{\epsilon}{2\left(X_{m_{1}}^{2}-X_{m_{2}}^{2}\right)}-\sqrt{\frac{\tilde{\alpha}\left(X_{m_{2}}^{2}-X_{m_{1}}^{2}\right)}{X\left(X_{m_{2}}^{2}+X_{m_{1}}^{2}\right)}}+O\left(\epsilon^{2}, \tilde{\alpha}\right)
\end{aligned}
$$

(In the above equations we assumed that $X_{m_{1}}^{2}>X_{m_{2}}^{2}$ and $\tilde{\alpha}<0$, which is the case in the numerical study of section 8. Similar equations can be derived when $X_{m_{1}}^{2}<X_{m_{2}}^{2}$ ).

The solution $t=t_{1}$ is thus the most relevant for the study of the behaviour of $u$ in the flatness limit, $\epsilon \rightarrow 0$. One finds,

$$
u\left(t_{1}\right) \sim \frac{X_{m_{2}}^{2}-X_{m_{1}}^{2}}{2 \tilde{\alpha}}+\epsilon \frac{X_{m_{1}}^{2}}{2 \tilde{\alpha}\left(X_{m_{1}}^{2}-X_{m_{2}}^{2}\right)}+O\left(\epsilon^{2}\right)
$$

which is enough to show that, as far as $\tilde{\alpha}$ is not vanishing (which is necessarily the case when $\tan \beta$ is close to 1 , see Eq.(4.2)), $u$ is not singular and $\zeta$ remains of order $\tilde{\alpha}$. 
The limiting behaviour of $u$ in Eqs.(4.28, 4.33) will allow also to give an explanation for the fact that the instability of the vev's in the vicinity of the D-flatness is much less pronounced at one-loop order than at tree-level, as was noted in [6]. We will come back to this point again in the numerical discussion of section 9 .

Let us now state some further properties which allow to determine the relative magnitudes of $\sqrt{t_{ \pm}}$as compared to those of $T_{ \pm}$and to $\frac{m_{t}}{m_{b}}$. $\zeta(t)$ has the nice property of being an increasing (resp. decreasing) function of $t$ when $\tilde{\alpha}>0$ (resp. $\tilde{\alpha}<0)$.

An immediate consequence of this and of the generic behaviour of $t_{ \pm}(\zeta)$ (see Fig. 1 ), is that the maximal value of $t_{+}$is $t_{+}^{\max }=t_{+}\left(\zeta\left(\frac{m_{t}}{m_{b}}\right)\right)$. By the same token, one has $t_{+}^{\min }=t_{+}\left(\zeta\left(T_{+}\right)\right), t_{-}^{\max }=t_{-}\left(\zeta\left(T_{-}\right)\right)$and $t_{-}^{\min }=t_{-}(\zeta(t \rightarrow 1))$. From this one can show that $\sqrt{t_{+}^{\text {min }}}$ can never get greater than $\tan \beta_{+}$whatever the sign of $\tilde{\alpha}$ is. This has the important consequence that the $t_{ \pm}$bounds alone cannot exclude the region $\tan \beta>1$, while the $T_{+}$bound can do so, viz. when $T_{+}>\frac{m_{t}}{m_{b}}($ with $\tilde{\alpha}<0)$ or $T_{+}<\frac{m_{t}}{m_{b}}($ with $\tilde{\alpha}>0)$. In contrast, one can determine at least sufficient conditions, for instance in the region $X_{m_{1}}^{2}+X_{m_{2}}^{2}+2 X_{m_{3}}^{2}<<1$, for which $\sqrt{t_{-}^{m i n}}<T_{-}$, thus implying that the $\tan \beta<1$ region is fully excluded in this case. Furthermore, these conditions can be turned into exclusion domains in the $\left(Y_{t}, Y_{b}\right)$ plane.

\section{The model-independent $\tan \beta$ bounds:}

Let us summarize here the new lower and upper bounds we found for $\tan \beta$. We rewrite them in a way which explicates the relevance of the sign of $\tilde{\alpha}$ and assume without loss of generality $X_{m_{3}}^{2}$ to be negative (see appendix A for a related discussion):

a) $\tilde{\alpha} \leq 0$

$$
\begin{gathered}
\text { if } \tan \beta>1 \text { then } T_{+} \leq \tan \beta \leq \frac{m_{t}}{m_{b}} \text { and } \tan \beta \geq \sqrt{t_{+}} \\
\text {if } \tan \beta<1 \text { then } T_{-} \leq \tan \beta<1 \text { and } \tan \beta \leq \sqrt{t_{-}}
\end{gathered}
$$

b) $\tilde{\alpha} \geq 0$

$$
\frac{m_{t}}{m_{b}} \leq \tan \beta \leq T_{+} \text {and } \tan \beta \geq \sqrt{t_{+}}
$$


where

$$
T_{ \pm}=\frac{-X_{m_{1}}^{2}-X_{m_{2}}^{2} \mp \sqrt{\left(X_{m_{1}}^{2}+X_{m_{2}}^{2}\right)^{2}-4 X_{m_{3}}^{4}}}{2 X_{m_{3}}^{2}}
$$

and

$$
t_{ \pm}=\frac{\tilde{\alpha}^{2} \frac{v_{1} v_{2}}{X_{m_{3}}^{2}}-X \mp \sqrt{\left(X-\tilde{\alpha}^{2} \frac{v_{1} v_{2}}{X_{m_{3}}^{2}}\right)^{2}+\tilde{\alpha}^{2}-X^{2}}}{\tilde{\alpha}-X}
$$

with $0<T_{-} \leq 1 \leq T_{+}$when the potential is bounded from below, and $0<t_{-} \leq 1 \leq t_{+}$ in all cases ( see Fig.1). Here we also

We also note that the slight dissimilarity between $\tan \beta<1$ and $\tan \beta>1$ in a) should not be seen as contradicting the invariance of the Lagrangian (and the effective action) under the substitutions top $\leftrightarrow$ bottom, $H_{2} \leftrightarrow H_{1}$. Indeed this invariance is easily seen to hold in Eqs.(4.1, 4.12 4.14), but the point is that $\tan \beta$ and $\cot \beta$ are not simultaneous solutions of equation (4.13), in contrast with the tree-level case where the two $\tan \beta$ solutions are inverse of each other, Eq.(3.9).

Excluding $\tan \beta=1$ : We close this section by remarking that $\tan \beta=1$ should be excluded as corresponding to a physically unsatisfactory situation. Actually the $t_{ \pm}$ bounds exclude generically a region around this value. Still, we want to stress here that $\tan \beta=1$ should be excluded on more general grounds. When $v_{1}=v_{2} \neq 0$, Eq. (4.12) implies $X_{m_{1}}^{2}+X_{m_{2}}^{2}+2 X_{m_{3}}^{2}=0$. The latter implies in turn that the effective potential Eq.(4.1) becomes flat ( $V=0$, barring of course a cosmological constant) everywhere along the direction $v_{1}=v_{2}$. The same is true at tree-level as was already remarked in section 3 . However, while at tree-level any point along the $\tan \beta=1$ was indeed a stationary point, this degeneracy is now lifted as can be easily seen from Eqs.4.12), and the only EWSB stationary point along this direction is given by

$$
v_{1}^{2}=v_{2}^{2}=\frac{X_{m_{2}}^{2}+X_{m_{3}}^{2}}{\tilde{\alpha}}=-\frac{X_{m_{1}}^{2}+X_{m_{3}}^{2}}{\tilde{\alpha}}
$$

The point is that this stationary solution is not stable and cannot be a minimum. One can see this easily in the particular case at study, from the fact that Eq.(4.17) is not satisfied when $v_{1}=v_{2}$. [Note that $\tilde{\alpha} \neq 0$ when $v_{1}=v_{2}$ since $\frac{m_{t}}{m_{b}}>>1$.] However we stress that there is a stronger reason which might suggest that the result continues to hold at higher orders of perturbation theory: given our approximate treatment of the logs as described in section 2, we find that to one-loop order the degeneracy is lifted but the flatness is maintained. The only way to go along with this is that the shape of the potential be twisted around the point given by Eq.(4.39). This means that this solution is a saddle-point rather than a minimum and should thus be excluded. 


\section{Meaning of the new bounds}

In this section we comment on the relation between the bounds we derived and some of the features in the existing literature. To start with, it should be clear that the $\tan \beta$ bounds a) and b) reflect but partially the constraints coming from Eqs.(4.12). This is why $X_{m_{1}}^{2}, X_{m_{2}}^{2}$ and $X_{m_{3}}^{2}$ are considered as three free parameters at this level. This means that the $\tan \beta$ bounds delimit the regions for which a stationary point is an actual (local) minimum with non-vanishing vev's. On the other hand, the $\tan \beta$ bounds contain the full information coming from the $2^{\text {nd }}$ order derivatives at the one-loop level Eqs.(4.17, 4.19), (keeping in mind that we absorbed the logarithmic terms in the tree-level part). They thus imply that the one-loop corrected Higgs mass-sum-rules, which have been extensively studied in the literature [17, 18, 19, 20], are such that the requirement of positivity of the squared Higgs masses leads in general to non trivial constraints. From this point of view, it is to be noted that if the one-loop minimum remains sufficiently close to its tree-level counterpart, then it is likely that these positivity conditions be automatic as is precisely the case at the tree-level. This was for instance the case in [17] where the D-terms in the stop mass matrix where neglected and the renormalization scale chosen such that the stationarity condition be fulfilled at the tree-level. The leading top-stop effect in the one-loop correction to the lightest CP-even Higgs squared mass turned out then to be always positive. More generally however, keeping those D-terms (which lead to the $\tilde{\alpha}$ contributions) one should rely on the one-loop stationarity condition, and as can be seen for instance from ref. [18] the positivity is no more automatic. Conditions a) and b) are the analytic constraints (within our approximation) which ensure this positivity.

Let us now compare briefly some of the well-known constraints, namely $1 \leq \tan \beta \leq$ $\frac{m_{t}}{m_{b}}$, and our condition a). We recall first that $1 \leq \tan \beta \leq \frac{m_{t}}{m_{b}}$ is a model-dependent result in the context of SUGRA-GUT [16] obtained through the running of $m_{1}^{2}$ and $m_{2}^{2}$ as dictated by the theory, in Eq.(3.16). Actually one can easily see from Eq.(3.17) and what precedes it, that in order to exclude $\tan \beta<1$ one needs either $m_{2}^{2}<0$ or $m_{1}^{2}>m_{2}^{2}$. The first of these inequalities is a sufficient but not necessary condition for electroweak symmetry breaking to take place, so that one also needs to consider the condition $m_{1}^{2}>m_{2}^{2}$ in order to exclude $\tan \beta<1$ in all cases. This is where, besides the running of $m_{1}^{2}$ and $m_{2}^{2}$, enters the universality assumption $m_{1}^{2}\left(M_{G U T}\right)=m_{2}^{2}\left(M_{G U T}\right)$ leading to $m_{1}^{2}>m_{2}^{2}$ at the electroweak scale. In our case we assume none of the above model-dependent features. Yet, though fully model-independent, our condition $1 \leq \operatorname{Max}\left(T_{+}, \sqrt{t_{+}}\right) \leq \tan \beta \leq \frac{m_{t}}{m_{b}}$ appears to be stricter than the previous one since it excludes a calculable domain around $\tan \beta=1$. There is of course no contradiction here; it only means that using the tree-level 
(actually tree-level-RGE-improved) minimization conditions together with some modeldependent assumptions is weaker than just using the model-independent one-loop minimization conditions. Of course, one can still make the same model-dependent assumptions on top of the one-loop minimization conditions we derived and get even stronger bounds. Also our bounds should be contrasted with the more qualitative ones, derived in the literature from the requirement of perturbativity of the Higgs-top or Higgs-bottom Yukawa couplings, [21]. It is clear that the requirement of perturbativity is more of practical than physical relevance, while our constraints are directly related to the physical requirement of symmetry breaking.

\section{Comments on necessity and sufficiency}

The constraints and bounds we established in section 4 are necessary for the existence of local minima which break $S U(2)_{L} \times U(1)_{Y}$ to $U(1)_{e . m}$. If there exists more than one such minimum, the true minimum will be of course the lowest, or at least a sufficiently metastable one. However, when it comes to assess the sufficiency of our conditions several other questions should be asked. First, does the symmetric vacuum become unstable as a consequence of the existence of an electroweak vacuum as was the case at tree-level (see Eqs. $(3.14,3.15))$ ? At vanishing $H_{1}$ and $H_{2}$, the invariants of the matrix of second order derivatives of the one-loop effective potential with respect to the eight components of $H_{1}$ and $H_{2}$, are all equal to

$$
\mathcal{I}=X_{m_{1}}^{2} X_{m_{2}}^{2}-X_{m_{3}}^{2}
$$

which is a direct generalization of the tree-level case, see Eqs.(3.14, 3.15). Substituting for $X_{m_{1}}^{2}$ and $X_{m_{2}}^{2}$ through Eq.(4.12) one finds

$$
\mathcal{I}=-\tilde{\alpha}^{2} v_{1}^{2} v_{2}^{2}+\left(v_{2}^{2}-v_{1}^{2}\right)\left[\tilde{\alpha}\left(v_{1}^{2}+v_{2}^{2}\right)-\left(v_{2}^{2}-v_{1}^{2}\right) X\right]\left(X-\frac{X_{m_{3}}^{2}}{v_{1} v_{2}}\right)
$$

Upon use of Eqs.(4.17, 4.19) one then shows easily that

$$
\mathcal{I} \leq \tilde{\alpha}^{2} v_{1}^{2} v_{2}^{2}\left(2 \frac{v_{1} v_{2}}{X_{m_{3}}^{2}} X-3\right) \leq 0
$$

Thus the one-loop conditions for the existence of EWSB local minima are sufficient to make the gauge symmetric vacuum unstable. It should be clear that this is not a self-evident statement. There would be nothing wrong with a local minimum at the gauge symmetric point, but then one would have had to impose extra constraints for this minimum not to be the global minimum. 
Up to now all the discussion was lead in the electrically neutral $H_{1}, H_{2}$ directions defined by Eq.(3.2). Charge breaking directions like

$$
H_{1}=\left(\begin{array}{c}
0 \\
H_{1}^{-}
\end{array}\right) \quad H_{2}=\left(\begin{array}{c}
0 \\
H_{2}^{0}
\end{array}\right)
$$

are usually assumed to be devoid of minima. Indeed, such minima do not occur at tree-level or "tree-level improved" approximation in the MSSM. They do however occur in a general two-higgs-doublet model, and imply in this case some constraints on the tree-level Higgs couplings [22]. Furthermore, knowing of no general proof guaranteeing the absence of such minima at higher loop orders even in the MSSM, we have to study the one-loop case explicitly. It is instructive to show briefly what the typical pattern of first and second order derivatives looks like and how it changes when one goes from tree-level to one-loop order. We will take for illustration the direction defined in Eq.(6.3), and assume furthermore $\operatorname{Im}\left(H_{1}^{-}\right)=\operatorname{Im}\left(H_{2}^{0}\right)=0$ for simplicity. We denote the vev's $<\operatorname{Re}\left(H_{1}^{-}\right)>$and $<\operatorname{Re}\left(H_{2}^{0}\right)>$ respectively by $c_{1}$ and $c_{2}$. Demanding that $c_{1}$ and $c_{2}$ be simultaneously non-vanishing requires $X_{m_{3}}^{2}=0$ and leads to the following stationarity conditions

$$
\begin{aligned}
& 2(X-\tilde{\alpha}) c_{2}^{2}+(\tilde{\beta}-2 X) c_{1}^{2}+2 X_{m_{2}}^{2}=0 \\
& 2(X+\tilde{\alpha}) c_{1}^{2}+(\tilde{\beta}-2 X) c_{2}^{2}+2 X_{m_{1}}^{2}=0
\end{aligned}
$$

The above equations are also valid at tree-level, the replacement of $\tilde{\alpha}, \tilde{\beta}, X, X_{m_{i}}^{2}$ by their tree-level counterparts being understood. Since $\tilde{\beta} \pm 2 \tilde{\alpha}$ is always perturbatively positive valued, summing Eqs.(6.4, 6.5) and taking into account the fact that $c_{1}^{2}$ and $c_{2}^{2}$ are positive, implies readily that $X_{m_{1}}^{2}+X_{m_{2}}^{2}<0$. The last inequality together with $X_{m_{3}}^{2}=0$ violates Eq.(4.11) and leads to an unbounded from below effective potential. If one takes the unboundedness from below of the MSSM as a disaster then it should be concluded that charge breaking minimum in the Higgs sector cannot occur in realistic situations. Indeed in sections 3 and 4 we assumed boundedness from below all the way. From this point of view, combining Eq. (6.2) and the absence of charge breaking just illustrated one concludes that the necessary EWSB conditions derived in those sections are also sufficient, at least in the Higgs fields directions.

From a more general point of view, though, one should keep open the possibility that the full theory can still be well behaved even in the case where the MSSM has an 
unbounded from below potential. After all, the MSSM is only a low-energy effective theory while the behaviour of the effective potential at very large field values becomes sensitive to the heavy $\left(\sim M_{G U T}\right)$ degrees of freedom whose interactions might cure the apparent unboundedness from below. In this case one should study further the second order derivatives to question the occurrence of charge breaking minima. An important difference shows up between the tree-level and one-loop cases, of which we give here just a glimpse. In both cases one finds only three independent invariants of the second order derivative matrix. At tree-level, two of these invariants have the following form:

$$
\begin{aligned}
\mathcal{C}_{1}^{\text {tree }} & =m_{1}^{2}+m_{2}^{2} \\
\mathcal{C}_{2}^{\text {tree }} & =-\frac{g_{1}^{2}+g_{2}^{2}}{g_{2}^{2}}\left(m_{1}^{2}+m_{2}^{2}\right)
\end{aligned}
$$

and are thus always of opposite signs irrespectively of the question of unboundedness from below. Thus at tree-level there is no way of having a (even local) charge breaking minimum. [Note also that $\mathcal{C}_{1}^{\text {tree }}=\mathcal{C}_{2}^{\text {tree }}=0$ would lead through Eqs.(6.4, 6.5) to zero charge breaking vev's.]

The situation is more dangerous at one-loop order. The three invariants read in this case

$$
\begin{aligned}
\mathcal{C}_{1}^{1-\text { loop }} & =\left(\mathcal{R}_{1}+\mathcal{R}_{2}\right) \frac{\tilde{\beta}}{4 \tilde{\alpha}^{2}+\tilde{\beta}^{2}-4 \tilde{\beta} X} \\
\mathcal{C}_{2}^{1-\text { loop }} & =-\frac{4 \mathcal{R}_{1} \mathcal{R}_{2}}{4 \tilde{\alpha}^{2}+\tilde{\beta}^{2}-4 \tilde{\beta} X} \\
\mathcal{C}_{3}^{1-\text { loop }} & =(\tilde{\alpha}-X)(2 \tilde{\alpha}-\tilde{\beta}+4 X) X_{m_{1}}^{2}+(\tilde{\alpha}+X)(2 \tilde{\alpha}+\tilde{\beta}-4 X) X_{m_{2}}^{2}
\end{aligned}
$$

where

$$
\begin{aligned}
& \mathcal{R}_{1}=2(\tilde{\alpha}-X) X_{m_{1}}^{2}+(\tilde{\beta}-2 X) X_{m_{2}}^{2} \\
& \mathcal{R}_{2}=(\tilde{\beta}-2 X) X_{m_{1}}^{2}-2(\tilde{\alpha}+X)
\end{aligned}
$$

and give back, of course, the tree-level results when $\tilde{\alpha} \rightarrow 0, \tilde{\beta} \rightarrow g_{2}^{2} / 2, X \rightarrow\left(g_{1}^{2}+g_{2}^{2}\right) / 8$ and $X_{m_{i}}^{2} \rightarrow m_{i}^{2}$. However one can find now a region, in the perturbative regime, where $\mathcal{C}_{1}^{1-\text { loop }} \geq 0, \mathcal{C}_{2}^{1-\text { loop }} \geq 0, \mathcal{C}_{3}^{1-\text { loop }} \geq 0$ are simultaneously verified and consistent with $X_{m_{1}}^{2}+X_{m_{2}}^{2} \leq 0$ which is required by Eqs. (6.4, 6.5). This happens if $|\tilde{\alpha}-X|, \tilde{\alpha}+X<\tilde{\beta}-2 X$. The point is that the perturbative realization of these last inequalities depends on the 
relative magnitudes of $g_{1}^{2}$ and $g_{2}^{2}$. For instance they are not satisfied at the unification scale where one has typically $g_{2}^{2}=(5 / 3) g_{1}^{2}$, but they are satisfied at the electroweak scale where $g_{2}^{2} \sim 3.3 g_{1}^{2}$. If anything, this means that, provided the apparent unboundedness from below is tolerated (see also the related comment at the end of section 8), local charge breaking minima can occur in the Higgs directions of the MSSM at the one-loop level! One would then have to require such minima to remain higher than the electroweak one and to be sufficiently short lived. We do not dwell further on these aspects here.

Before closing this section we should note that one has in principle to study the stationarity and stability of the electroweak minimum in all squark/slepton directions as well. These aspects will be considered elsewhere. We will be contented here just with the remark that at tree-level the curvature of the effective potential is, on one hand, proportional to the squared squark (or slepton) masses in the respective squark (or slepton) directions at the zero fields point, and on the other, that this curvature remains positive in these directions also at the electroweak point, for realistic values of $m_{1}, m_{2}$ and squark/slepton masses. This does not mean, though, that no charge or color breaking minima can occur, since one can still be driven around a hump into a minimum in a charged or colored direction.

\section{Gauge-fixing dependence}

In this section, we make a short digression and discuss briefly the issue of gauge-fixing dependence of the constraints established so far. It is well-known that the effective potential $V_{\text {eff }}$ is generally a gauge-fixing dependent object, even though it is formed of gauge invariant operators. However, the values of $V_{\text {eff }}$ at stationary points, corresponding to average energy densities of "vacuum" states, are themselves gauge-fixing independent at least for a given class of gauges [23]. [This result holds perturbatively in the loop-wise expansion]. It follows that the condition $\frac{\partial V_{e f f}}{\partial \phi}=0$ selects physical stationary points, even though $\frac{\partial V_{\text {eff }}}{\partial \phi}$ as well as the field values at which it vanishes (including the vev's), are generically gauge-fixing dependent.

We want to point out hereafter that, under fairly general assumptions about $V_{\text {eff }}$, a (local) minimum or maximum, remains so for any value of the gauge-fixing parameter, even if the magnitude of $\frac{\partial^{2} V_{e f f}}{\partial \phi^{2}}$ is itself gauge-fixing dependent.

Let us assume that $V_{\text {eff }}(\phi, \xi)$ is a smooth continuous function of $\xi$, where $\xi$ is a generic gauge parameter, and that $V_{\text {eff }}$ is bounded from below. As far as boundedness from below is concerned, one can think of this condition as requiring something like Eq.(4.11) simul- 
taneously for any value of the $\xi$ parameter 11 . We can now show that a (local) minimum for a given value $\xi_{1}$ of the gauge parameter, cannot transform into a (local) maximum or a saddle-point for another value $\xi_{2}$. Indeed, since $V_{\text {eff }}$ is $\xi$-independent at every stationary point [23], the only way of changing a (local) minimum at a point $\mathrm{M}$, when $\xi_{1}$ goes to $\xi_{2}$, is to create a new stationary point M' which corresponds to a lower value of $V_{\text {eff }}$, and which lies between $\mathrm{M}$ and the next nearby stationary point. However, this new stationary point (which exists for $\xi_{2}$ and not for $\xi_{1}$ ) must be degenerate with one of the already pre-existing stationary points at the value $\xi_{1}$, otherwise it would contradict the $\xi$-independence of $V_{\text {eff }}$ at stationary points. But even if such a degeneracy occurs, $V_{\text {eff }}$ being a smooth continuous function of $\xi$ there will always be a value of $\xi$ between $\xi_{1}$ and $\xi_{2}$ for which a new stationary point appears and is not (yet) degenerate with any of the previously existing ones, thus contradicting the aforementioned $\xi$-independence. This completes the "proof" that if $\frac{\partial^{2} V_{e f f}}{\partial \phi^{2}}>0$ at stationary points for a given value of $\xi$, then it is fulfilled for any value of $\xi$. A similar reasoning applies when there are more than one scalar field, and also in the case of (local) maxima or saddle-points. We illustrate in Fig.2 a typical behaviour of the effective potential under a gauge-fixing change.

The above discussion shows that the perturbative determination of the conditions for the occurrence of (local) minima is physically meaningful, despite the $\xi$-dependence of the quantities we compute (i.e. first and second order derivatives). Nonetheless, the fact that the vev's are $\xi$-dependent requires some precaution when relating them to the (physical) electroweak scale. For instance, when $M_{Z}^{2}$ is equated to $\sim\left(g_{1}^{2}+g_{2}^{2}\right)\left(v_{1}^{2}+v_{2}^{2}\right)$ to obtain Eqs.(3.1) as the condition of symmetry breaking at the correct electroweak scale, one should keep in mind that this is but a gauge-fixing dependent approximation. The physical mass $M_{Z}$ is the mass pole which is not given (beyond the tree-level) by the quantity $\left(g_{1}^{2}+g_{2}^{2}\right)\left(v_{1}^{2}+v_{2}^{2}\right)$, the latter being $\xi$-dependent, [23].

\section{Numerical illustration}

The one-loop EWSB equations and constraints established in section 4 should be very helpful in the implementation of any radiative symmetry breaking study in the MSSM. They are fully model independent, and must be adapted in each model which triggers electroweak symmetry breaking. In this section we give a very first illustrative example in a SUGRA-GUT context. Since the aim is just to compare the tree-level and one-loop nonlogarithmic contributions, we will take up here, as discussed in section 2, the simple (and

\footnotetext{
${ }^{11}$ This avoids questions related to whether the necessary and sufficient boundedness from below conditions are gauge-fixing dependent or not.
} 
somewhat old fashioned) approximation of the renormalization group improved tree-level, without taking into account supersymmetric threshold effects which would not however modify the qualitative picture.

We show how the various bounds and constraints presented in section 4 conspire to give, as in the the tree-level case, only one local minimum of the potential. This should not come as a surprise. At the tree-level, the equation for $\tan \beta$ is quadratic and gives at most two real solutions. Actually, we fall in the situation depicted in section 3, where one solution gives $0<\tan \beta<1$. Together with $m_{2}^{2}<0$, this solution is thus rejected for $u \equiv v_{1} v_{2}$ becomes negative. This situation is common in SUGRA-GUT motivated analyses. Universality for the soft scalar mass terms combined with the relative rapid decrease with the scale of $m_{2}{ }^{2}$ in comparison with $m_{1}^{2}$ ( due to the contribution of $Y_{t}$ in its renormalization group equation) drives $m_{2}^{2}$ negative while $m_{1}^{2}$ remains positive. By tuning initial values at GUT scale, we can arrange to have $m_{1}{ }^{2} m_{2}{ }^{2}-m_{3}{ }^{2}<0$ near electroweak scale. This is the signal that the gauge-invariant vacuum is unstable, leading to the electroweak symmetry breaking ( we discard here the possibility of CCB minima outside the higgs direction). Now adding to the tree-level potential one-loop non-logarithmic contributions, the equation for $\tan \beta$ Eq. (4.13) becomes quartic. So we can potentially have up to four different and real solutions in this case. However all of them do not correspond to local minima, and the bounds established in section 4 are precisely here to reject all of them but one which is near the local minimum obtained at the tree-level.

This is not the only point of the discussion. $\tilde{\alpha}$ also contributes to moderate the behaviour of $v_{1}$ and $v_{2}$ as compared to the well-known tree-level wild behaviour near the scale where the potential becomes unbounded from below. We will also show how this comes about. Assuming universality at some unification scale $\tilde{\alpha}, X$, and the $X_{m_{i}}^{2}$ 's can be expressed as follows, see Eqs.(4.2-4.8)

$$
\begin{aligned}
\tilde{\alpha}= & \frac{12}{5} \kappa g_{0}^{2}\left(Y_{t}^{0^{2}}-Y_{b}^{0^{2}}\right) \\
X= & \frac{\bar{g}_{1}^{2}\left(\mu_{R}\right)+\bar{g}_{2}^{2}\left(\mu_{R}\right)}{8}+\kappa g_{0}^{2}\left(\frac{367}{100}-\frac{12}{5}\left(Y_{t}^{0^{2}}+Y_{b}^{0^{2}}\right)\right) \\
X_{m_{1}}^{2}= & \bar{m}_{1}^{2}\left(\mu_{R}\right)+\kappa\left[\frac{72}{15} g_{0}^{2} m_{1 / 2}^{2}+\frac{18}{15}\left(m_{0}^{2}-3 \mu_{0}^{2}\right)\right. \\
& \left.+12\left(\mu_{0}^{2} Y_{t}^{0^{2}}+Y_{b}^{0^{2}}\left(A_{b}^{0^{2}}+2 m_{0}^{2}\right)\right)\right] \\
X_{m_{2}}^{2}= & \bar{m}_{2}^{2}\left(\mu_{R}\right)+\kappa\left[\frac{72}{15} g_{0}^{2} m_{1 / 2}^{2}+\frac{18}{15}\left(m_{0}^{2}-3 \mu_{0}^{2}\right)\right. \\
& \left.+12\left(\mu_{0}^{2} Y_{b}^{0^{2}}+Y_{t}^{0^{2}}\left(A_{t}^{0^{2}}+2 m_{0}^{2}\right)\right)\right] \\
X_{m_{3}}^{2}= & \bar{m}_{3}^{2}\left(\mu_{R}\right)+\kappa \mu_{0}\left(\frac{18}{15}\left(B_{0}+4 m_{1 / 2}\right)-12\left(A_{t}^{0} Y_{t}^{0^{2}}+A_{b}^{0} Y_{b}^{0^{2}}\right)\right)
\end{aligned}
$$


We run the tree-level part of the above equations, using the one-loop RGE solutions for small $\tan \beta$, and keep their one-loop parts fixed at their initial values. Here $g_{0}$ is the unified value of the gauge couplings (taking a grand unified normalization for the $U(1)_{Y}$ coupling, $\left.g_{0}^{2}=g_{2}^{0^{2}}=5 / 3 g_{1}^{0^{2}}\right)$. We take for illustration, neglecting the contribution of $Y_{b}^{0}$, the following values of universal soft masses and other parameters which correspond to a no-scale scenario.

$$
\begin{aligned}
& A_{0}=B_{0}=m_{0}=0, \quad m_{\frac{1}{2}}=50 \mathrm{GeV}, \quad \frac{g_{0}^{2}}{4 \pi}=\frac{1}{24.5} \\
& \mu_{0}=-105 \mathrm{GeV}, \quad \frac{\left(Y_{t}^{0}\right)^{2}}{16 \pi^{2}}=0.01, \quad Y_{b}^{0} \sim 0, \quad M_{G U T}=2 \times 10^{16} \mathrm{GeV}
\end{aligned}
$$

In this illustration, because $\tilde{\alpha}$ is purely a one-loop effect, it will not vary with the renormalization scale $\mu_{R}$. The initial values above give a negative $\tilde{\alpha}, \tilde{\alpha} \sim-4.6 \times 10^{-3}$. Near the electro-weak scale, where we will focus our attention in the following, we also find $X_{m_{3}}^{2}$ to be negative, giving thus positive $T_{+}$and $T_{-}$( $\operatorname{sign}$ opposite to the one of $X_{m_{3}}^{2}$ ). Varying the scale $\mu_{R}$ around the electro-weak scale, we then solve the quartic equation in $\tan \beta$ (4.13). It should be clear that all the solutions (as well as the corresponding vev's) will vary with $\mu_{R}$. In our illustration, all of them are real, and for convenience we may label each one by comparing with the limiting case $\tilde{\alpha}=0$ where the solutions are just $T_{+}$, $T_{-}, 1$ and -1 . But one must keep in mind that, as soon as $\tilde{\alpha} \neq 0, T_{+}$and $T_{-}$are no more solutions, but rather bounds.

All the solutions do not correspond to local minima. In fact, we can safely discard the solution which is near -1 , because being negative, it contradicts constraint (4.19). The solution near $T_{-}$is rejected for the reason that it is included in $\left[0, T_{-}\right]$and so contradicts constraint (4.35). We must also reject the solution near 1 , because it is included in $\left[\sqrt{t_{-}}, \sqrt{t_{+}}\right]$, and does not verify constraints (4.34-4.35). We call this last solution $t_{1}$, for it coincides with Eq.(4.30) in the flat limit. Fig.3 shows its sensitivity to the renormalization scale and displays its theoretical bounds.

Note that to reject all those solutions, we have fully used constraints $(4.19,4.34,4.35)$ which contain all the qualitative informations on the second stationarity equation (4.14). They also give information on the stability of the possible extrema. If we had used only the two stationarity equations, we could have already rejected the first two solutions since the corresponding $u\left(\equiv v_{1} v_{2}\right)$ would have had a sign opposite to that of $\tan \beta$. The third solution, $t_{1}$, would not be correctly rejected this way, being indeed an extremum of the 
potential but not a local minimum.

Finally, there is only one solution that will satisfy all constraints. This is the one near $T_{+}$ which we call $t_{2}$ for it gives Eq.(4.31) in the flat limit. It is such that $\mathrm{u}$ and $t_{2}$ have the same sign, and so we can be sure that it indeed corresponds to a local minimum of the potential. We are thus left, as in the tree-level case, with only one correct electro-weak minimum.

Fig.4 displays the variation with respect to $\mu_{R}$ of $t_{2}$ and its theoretical bounds. For comparison, we also give in the same figure the variation of the tree-level solution $\tan \beta_{0}$.

In the bounded from below region, that is above the scale $\mu_{R}^{U F B} \sim 52 \mathrm{GeV}$ (defined by $\left.X_{m_{1}}^{2}+X_{m_{2}}^{2}+\left.2 X_{m_{3}}^{2}\right|_{\mu_{R}^{U F B}}=0\right), t_{2}$ is just above the bound $T_{+}$and follows it as the scale decreases. It is also far above $\sqrt{t_{+}}$which is very close 1 . Generically, we have this hierarchy in SUGRA-motivated analysis: $\sqrt{t_{+}}$is never greater than $T_{+}$, except in a small region above $\mu_{R}^{U F B}$. Note also that $\tan \beta_{0}$ is below $T_{+}$and so does not verify the constraints (4.34). In fact, there is no reason that it should. The only lower bound we may give at the tree-level is $\tan \beta_{0}>1$ for $m_{2}^{2}<0$. We just want to stress here that the new stability constraints $T_{+}$can be quite significant and forbid some values of $\tan \beta$ including large ones taken by $\tan \beta_{0}$.

$T_{+}$and $T_{-}$terminate at $\mu_{R}^{U F B}$ and take at this scale the common value 1 , while $t_{2}$ ( and so does $t_{1}$, see Fig.3) continues to exist and still makes sense at and below $\mu_{R}^{U F B}$. This situation is quite typical of the one-loop non-logarithmic contributions. On the contrary, the tree-level solution $\tan \beta_{0}$ terminates when the unboundedness from below scale $\left(\mu_{R}^{U F B}\right)_{\text {tree }} \sim 54 \mathrm{GeV}$ ( defined by $m_{1}^{2}+m_{2}^{2}+\left.2 m_{3}^{2}\right|_{\left(\mu_{R}^{U F B}\right)_{t r e e}}=0$ ) is reached.

Finally, in the UFB region where $T_{+}$and $T_{-}$no more exist, the bounds $\sqrt{t_{+}}$and $\sqrt{t_{-}}$take over from them. As long as $t_{2}$ remains above $\sqrt{t_{+}}$in Fig.4, the corresponding stationary point is indeed a local minimum with finite vev. There is a critical scale where $t_{2}$ meets $\sqrt{t_{+}}$. If we look back in Fig.3, we see that this phenomenon also appears for $t_{1}$, at the same scale and with the same value $t_{1}=t_{2} \sim 1.12$. In this case the extremum could become a local minimum of the potential and we may wonder if, below the common critical scale, $t_{1}$ could take over on $t_{2}$. In fact, this does not happen: both $t_{1}$ and $t_{2}$ stop to be real valued, and this is the reason why, in Fig.3-4, the curves $t_{1}$ and $t_{2}$ suddenly stop. This behaviour of the solutions in $\tan \beta$ of Eq. (4.13) is typical of quartic equations, and always takes place in the UFB region.

Fig.5 gives the variation with respect to $\mu_{R}$ of the vev $v_{1}$ and $v_{2}$ associated with the tree-level solution $\tan \beta_{0}$ and with the one-loop solution $t_{2}$.

A few comments are in order so as to compare the tree-level approximation with the oneloop non-logarithmic one. The curves obtained are essentially similar when the potential 
is bounded from below, but behave quite differently in each approximation near the UFB region. This point is of course linked to the preceding discussion on the $\tan \beta$ solutions. At the tree-level, $v_{1}$ and $v_{2}$ blow up in the vicinity of $\left(\mu_{R}^{U F B}\right)_{\text {tree }}$ which is reached only asymptotically and never crossed. This well-known wild behaviour depends on the values taken by the mass parameters that enter the tree-level potential near this scale. A quick look at the extremal equations Eq. $(3.3,3.4)$ shows that $u$ remains finite if $\tan \beta=1$ only for particular values of the mass parameters. We must realize $m_{1}^{2}+m_{2}^{2}+2 m_{3}^{2}=0$ with the requirement that $m_{1}^{2}=m_{2}^{2}=-m_{3}^{2}$. In a SUGRA-GUT context, this is obviously impossible, and even if we deviate from universality for the soft scalar masses, this last condition should be adjusted by a fine-tuning which seems rather unnatural. In our illustration, at $\left(\mu_{R}^{U F B}\right)_{\text {tree }}$ we have $m_{1}^{2} \sim 6457 \mathrm{GeV}, m_{2}^{2} \sim-3133 \mathrm{GeV}, m_{3}^{2} \sim-1662 \mathrm{GeV}$ which makes $m_{1}^{2}+m_{2}^{2}+2 m_{3}^{2} \sim 0$. The tree-level potential becomes flat and vanishing in the direction $v_{1}=v_{2}$ and $\tan \beta=1$ is the only solution toward which $\tan \beta_{0}$ is attracted. But the condition $m_{1}^{2}=m_{2}^{2}=-m_{3}^{2}$ is not fulfilled, so the flat direction will be reached only asymptotically with exploding $v_{1}$ and $v_{2}$.

In contrast, Fig.5 shows that in the one-loop non-logarithmic approximation, $v_{1}$ and $v_{2}$ do not have a singular behaviour near $\mu_{R}^{U F B}$. We can understand this point by noting that $t_{2}$ is not attracted to 1, (see Eq.(4.31) and Fig.4), so u remains finite.[Note however that $\tan \beta=1$ is not a singular value for $\mathrm{u}$, as can be seen in Eq.(4.33), because of the genuine effect of $\tilde{\alpha}$, whatever its magnitude.] Although the vev's vary rapidly near the "critical scale" (where $t_{2}$ stops to exist), they still remain finite. Below this scale the vev's become complex valued, and disappear in Fig.5.

Regarding the mild behaviour of the vev in comparison with the tree-level one, we can say that adding non-logarithmic terms to the tree-level potential, even before including logarithmic ones, already contributes to stabilize the vev of the Higgs fields.

Remarks on unboundedness from below One might inquire whether it is physically relevant to consider, as we did in the previous paragraph, the regions of the parameter space where the effective potential is unbounded from below. Considering the MSSM as a low-energy approximation of a more fundamental theory which would set in at high energy (presumably $M_{G U T}$ ), one should keep in mind that our effective potential is also "effective" in the sense that only light degrees of freedom are taken into account. The fact that we consider regions where equation (4.11) is violated means that we implicitly assume the heavy degrees of freedom of the full theory to play an important role in lifting the effective potential at very large values $\left(\sim M_{G U T}\right)$ of the scalar fields and restore a bounded from below potential. In this case, the relevant question one can ask from a model-independent point of view is whether local minima exist even when $V_{\text {eff }}$ is a 
decreasing, and negative valued function at large field values, and whether any of these minima can be lower than the value of $V_{\text {eff }}$ for fields just below the scale where the full theory should set in.

\section{Conclusion}

We have demonstrated in this paper, the existence of fully model-independent constraints involving $\tan \beta$ and the other free parameters of the MSSM. These constraints are derived from a careful study of the EWSB conditions, including the finite (non-logarithmic) one-loop corrections to the effective potential. We have shown with this respect that stationarity and stability conditions ( which are necessary and sufficient to ensure the existence of a local minimum) are not automatically related beyond the tree-level approximation. We then determined the exact analytic forms of both and deduced new bounds on $\tan \beta$. These bounds are, generally speaking, resemblant to the existing ones, but they turn out to be more restrictive even though fully model-independent! They also distinguish quite naturally the "large" and "low" $\tan \beta$ regimes, restricting further the allowed range within each of these regimes.

As a first step, we resorted in this paper to the approximation where the logarithmic contributions are taken into account through the RG-improved tree-level potential. This, we believe, contains already many of the qualitative features of the problem at hand. The inclusion of the logarithmic effects beyond the present approximation will be presented elsewhere.

Finally, it is clear that the existence of model-independent constraints is interesting both theoretically and phenomenologically. For instance we noted that the connection between stability and stationarity, which is lost beyond the tree-level, can be partially preserved if $1<\tan \beta<\frac{m_{t}}{m_{b}}$. If this connection is a desirable theoretical criterion then the model-independent study helps select the model assumptions likely to satisfy it. More down to earth, given the variety of theoretical models and the fact that recent data seem to favour some of the MSSM parameter values that are not consistent with the conventional model assumptions [24], the model-independent constraints would help narrow down the domains that should be common features to all models. In particular our constraints to one-loop order are readily applicable in the context of R-parity violation, and can be generalized to the next to minimal MSSM. 


\section{Acknowledgments}

We would like to thank Nikolai Krasnikov for asking the question which lead to section

7. We also acknowledge useful discussions with Michel Capdequi-Peryranere, Abdelhak Djouadi and Jean-Loïc Kneur. 


\section{Appendix}

\section{A: $\quad$ The $\tan \beta$ bounds}

Starting from Eq. (4.14),

$$
u=\frac{1}{\tilde{\alpha}\left(t^{2}-1\right)} a(t)
$$

with $u \equiv v_{1} v_{2}, t \equiv \tan \beta$ and

$$
a(t)=X_{m_{3}}^{2}\left(t^{2}+1\right)+\left(X_{m_{1}}^{2}+X_{m_{2}}^{2}\right) t
$$

and assuming boundedness from below Eq (4.11), we note first that the two zeros of $a(t)$, i.e.

$$
T_{ \pm}=\frac{-X_{m_{1}}^{2}-X_{m_{2}}^{2} \mp \sqrt{\left(X_{m_{1}}^{2}+X_{m_{2}}^{2}\right)^{2}-4 X_{m_{3}}^{4}}}{2 X_{m_{3}}^{2}}
$$

have the same sign, opposite to that of $X_{m_{3}}^{2}$. Furthermore one has

$$
\left|T_{-}\right|<1<\left|T_{+}\right|
$$

the degenerate value 1 being attained when the inequality Eq.(1.11) is saturated. On the other hand, we note that

$$
\tilde{\alpha}<0 \Leftrightarrow\left|Y_{t}\right|>\left|Y_{b}\right| \Leftrightarrow|\tan \beta|<\frac{m_{t}}{m_{b}}
$$

as easily seen from Eqs. (4.2) and (4.9) and from the usual relations $m_{t}=\frac{\left|Y_{t} v_{2}\right|}{\sqrt{2}}, m_{b}=\frac{\left|Y_{b} v_{1}\right|}{\sqrt{2}}$. We keep here absolute values on purpose (and somewhat puristically), since we would like to establish clearly the freedom in choosing the $\operatorname{sign}$ of $\tan \beta$, which is rather tricky in the present context.

In the following we will address the question of whether the necessary stability condition $\frac{X_{m_{3}}^{2}}{v_{1} v_{2}} \leq 0$ (Eq.(4.19) ) is automatically fulfilled, and will establish in the process some model-independent analytic bounds on the allowed values of $\tan \beta$. The properties of $T_{ \pm}$ presented above, and the sign of $\tilde{\alpha}$ will be crucial for the subsequent discussion.

Regarding Eq.(A.5) we should consider the two cases

$$
|\tan \beta| \leq \frac{m_{t}}{m_{b}} \quad \text { and } \quad|\tan \beta| \geq \frac{m_{t}}{m_{b}}
$$

and in the former case, we study separately $\tan ^{2} \beta>1$ and $\tan ^{2} \beta<1$. 
Case 1) $|\tan \beta| \leq \frac{m_{t}}{m_{b}},(\tilde{\alpha}<0)$

(1-a) $\tan ^{2} \beta>1$ implies $\tilde{\alpha}\left(t^{2}-1\right)<0$. It follows that in Eq. (A.1]), $a(t)$ and $u$ [and thus $t]$ have opposite signs. This means that if $X_{m_{3}}^{2} \leq 0$ then

$$
\tan \beta \geq T_{+} \geq 1
$$

is the only allowed region. Indeed, on one hand,

$$
0 \leq T_{-} \leq \tan \beta \leq T_{+}
$$

would imply that $a \geq 0$, i.e. of the same $\operatorname{sign} \operatorname{as} \tan \beta$, and on the other hand, if $\tan \beta \leq T_{-}$ (which means that $\tan \beta \leq-1$, since $\tan ^{2} \beta>1$ and $T_{-} \leq 1$ ) then $a<0$. Thus again $a$ and $\tan \beta$ would have the same sign, which is forbidden.

Similarly, if $X_{m_{3}}^{2} \geq 0$ then

$$
\tan \beta \leq T_{+} \leq-1
$$

is the only possibility compatible with the requirement that $a$ and $\tan \beta$ be of opposite sign.

To summarize, one has

$$
\begin{aligned}
1 \leq T_{+} \leq \tan \beta & \text { if } \quad X_{m_{3}}^{2}<0 \\
\tan \beta \leq T_{+} \leq-1 & \text { if } \quad X_{m_{3}}^{2}>0
\end{aligned}
$$

and hence in both cases $\frac{X_{m_{3}}^{2}}{v_{1} v_{2}} \leq 0$ is automatically satisfied, so that the "tree-level logic" continues to hold at one-loop in this case.

(1-b) $\tan ^{2} \beta<1$; the proof goes along similar lines as before, except that now $a$ and $\tan \beta$ should be of the same sign. In this case, the allowed regions are,

$$
\begin{aligned}
1 \geq \tan \beta \geq T_{-} \geq 0 \quad \text { if } & X_{m_{3}}^{2}<0 \\
-1 \leq \tan \beta \leq T_{-} \leq 0 & \text { if } \quad
\end{aligned}
$$

Nonetheless, there is in this case a difference from the previous one, namely that the stability condition is not automatic in the region $-1 \leq \tan \beta \leq 0$ when $X_{m_{3}}^{2}<0$ and similarly in the region $0 \leq \tan \beta \leq 1$ when $X_{m_{3}}^{2}>0$. Both regions are actually not excluded by the stationarity condition Eq.(A.1) alone, and one has to make explicit 
reference to the stability condition Eq.(4.19). Strictly speaking, one should still ask whether the remaining stability condition Eq.(4.13) can guarantee $\frac{X_{m_{3}}^{2}}{v_{1} v_{2}} \leq 0$. We have actually exhibited numerical examples where it does not. Thus in this case, the "treelevel logic" does not go over to the one-loop level.

Now if we compare the two cases of Eqs. A.7) and Eqs. A.8), we see that no information is lost if one fixes the sign of $X_{m_{3}}^{2}$ from the start (by redefining, when needed, the phase of one of the two higgs fields in Eq.(4.1)). We still insist however, that this choice is tricky, since, in any case, Eq.(4.13) is likely to give $\tan \beta$ solutions with opposite signs ( in contrast with the tree-level case where the two solutions had the same sign, see Eq.(3.9) ). It would then be erroneous to choose invariably the positive $\tan \beta$ solutions. Rather, one should keep a handle on the actual sign of $X_{m_{3}}^{2}$ (corresponding to a given set of values of the MSSM parameters) and choose the $\tan \beta$ solution which satisfies Eq.(4.19). Once the right solution is determined, one is at liberty to redefine its sign and accordingly that of $X_{m_{3}}^{2}$. In practice, the wrong choice would manifest itself through negative $v_{1}^{2}, v_{2}^{2}$, when $|\tan \beta|>1$.

Case 2) $|\tan \beta| \geq \frac{m_{t}}{m_{b}},(\tilde{\alpha}>0)$. In this case $\tilde{\alpha}\left(t^{2}-1\right)>0$ so that $a(t)$ and $t$ must have the same sign. The allowed regions are then found to be

$$
\begin{aligned}
\frac{m_{t}}{m_{b}} \geq \tan \beta \geq T_{+} \quad \text { if } \quad & X_{m_{3}}^{2}<0 \\
T_{+} \leq \tan \beta \leq-\frac{m_{t}}{m_{b}} \quad \text { if } & X_{m_{3}}^{2}>0
\end{aligned}
$$

provided of course that

$$
\left|T_{+}\right| \leq \frac{m_{t}}{m_{b}}
$$

Note that, similarly to case (1-a), the stability condition $\frac{X_{m_{3}}^{2}}{v_{1} v_{2}} \leq 0$ is not automatically satisfied through the sign requirements of $a$ and $t$, and has to be explicitly used to exclude the regions $\tan \beta \leq-\frac{m_{t}}{m_{b}}$ (resp. $\tan \beta \geq \frac{m_{t}}{m_{b}}$ ) when $X_{m_{3}}^{2}<0$ (resp. $X_{m_{3}}^{2}>0$ ). Thus in this case too, the "tree-level logic" does not go over to the one-loop level. Finally, the same liberty in the choice of the sign of $X_{m_{3}}^{2}$ noted in case 1) above, holds here as well. 


\section{B: The method of invariants}

This method allows to determine simple positivity conditions of the eigenvalues of the squared mass matrix, without computing these eigenvalues. The invariants of the matrix $M$ are defined as the coefficients of $\lambda$ in the following (finite order) expansion,

$$
\begin{aligned}
& \operatorname{Det}(1+\lambda M)=e^{\operatorname{Tr} \ln (1+\lambda M)} \equiv 1+\sum_{i=1}^{\operatorname{dim} M} \operatorname{In} v_{i} \lambda^{i} \\
& \operatorname{Inv} v_{1}=\operatorname{tr}(M) \\
& \operatorname{Inv}_{2}=\frac{1}{2}\left(\operatorname{tr}\left(M^{2}\right)-\operatorname{tr}(M)^{2}\right) \\
& \text { etc... } \\
& I n v_{\operatorname{dim} M}=\operatorname{Det} M
\end{aligned}
$$

One can show that a sufficient and necessary condition for all the eigenvalues to be positive is that all the above invariants be positive. The nicety of this property is that

whatever the size of $M$ is, one can always determine analytically the positivity conditions of its eigenvalues by computing straightforwardly powers of traces of powers of M! 


\section{References}

[1] L.E. Ibañez and G.G. Ross, Phys. Lett. B110 (1982) 215; K.Inoue, A. Kakuto, H. Komatsu and S. Takeshita, Prog. Theor. Phys. 68 (1982) 927; 71 ( 1984) 413; L. ALvarez-Gaumé, M. Claudson and M.B. Wise, Nucl. Phys. B207 (1982) 96; J. Ellis, D.V. Nanopoulos and K. Tamvakis, Phys. Lett B121 (1983) 123; L.E. Ibañez, Nucl. Phys. B218 (1983) 514; L. ALvarez-Gaumé, J. Polchinski and M.B. Wise, Nucl. Phys. B221 (1983) 495; J. Ellis, J.S. Hagelin, D.V. Nanopoulos and K. Tamvakis, Phys. Lett. B125 (1983) 275; L.E. Ibañez and C. Lopez, Phys. Lett. B126 (1983) 54; Nucl. Phys. B236 (1984) 438; For a pedagogical review, see for instance R. Arnowitt and P. Nath, Lecture at Swieca School, Campos do Jordao, Brazil, Jan. 1993, and references therein;

[2] A.H Chamseddine, R.Arnowitt and P.Nath, Phys.Rev.Lett. 49 (1982) 970; R.Barbieri, S.Ferrara and C.A Savoy, Phys.Lett. B119 (1982) 343; L.Hall, J.Lykken and S.Weinberg, Phys.Rev. D27 (1983) 2359;

[3] M.Dine and A.E.Nelson, Phys.Rev.D48 (1993) 1277;

[4] see for instance J.A. Casas, A. Lleyda, C. Muñoz, Nucl. Phys. B471 (1995) 3, and references therein;

[5] D.J. Castaño, E.J. Piard and P. Ramond, Phys.Rev.D49 (1994) 4882; W. de Boer, R. Ehret and D.I. Kazakov, Z. Phys. C67 (1994) 647; V. Barger, M.S. Berger and P. Ohmann, Phys.Rev.D49 (1994) 4908;

[6] G. Gamberini, G. Ridolfi, F. Zwirner, Nucl. Phys. B 331 (1990) 331;

[7] see [22] and the first ref. in [5];

[8] G. Moultaka, hep-ph/9705330, (Talk given at the 32nd Rencontres de Moriond: Electroweak Interactions and Unified Theories, Les Arcs, France, 15-22 Mar 1997, to appear in the proceedings.) and C. Le Mouël, G. Moultaka, hep-ph/9708368 (talk given at the EEC Network meeting Tests of Electroweak Symmetry Breaking, Ouranoupolis, Greece, May 27 - June 1, 1997, to appear in the proceedings)

[9] C. Le Mouël, Ph.D. thesis, in preparation;

[10] B. de Carlos and J.A Casas, Phys. Lett. B309 (1993) 320; R. Arnowitt and Pran Nath, Phys. Rev. D46, 9 ,(1992) 3981; 
[11] C. Ford, D.R.T. Jones, P.W. Stephenson, M.B. Einhorn Nucl. Phys. B 395 (1993) 17; M. Bando, T. Kugo, N. Maekawa, H. Nakano, Phys. Lett. B 301 ( 1993) 83 and Prog.Theor.Phys.90 (1993) 405;

[12] M. Bando, T. Kugo, N. Maekawa, H. Nakano, Mod. Phys. Lett. A7 (1992) 3379;

[13] S. Coleman, E. Weinberg, Phys. Rev. D 7 (1973) 1888;

[14] For a review of the MSSM and related topics see H. P. Nilles, Phys. Rep. 110(1984)1; H. E. Haber and G. L. Kane, Phys. Rep. 117(1985)75; A. B. Lahanas and D. V. Nanopoulos, Phys. Rep. 145(1987)1;

[15] Supersymmetry and Supergravity by J. Wess and J. Bagger, Princeton Series in Physics;

[16] G.F. Giudice, G. Ridolfi, Z. Phys. C 41 ( 1988) 447; see also F. Zwirner in "Physics and Experiments in Linear Colliders", Saariselkä, Finland Sept.91, Eds. R. Orava, P. Eerola, M. Nordberg;

[17] J.Ellis, G. Ridolfi, F. Zwirner, Phys. Lett. B257 (1991) 83;

[18] J.Ellis, G. Ridolfi, F. Zwirner, Phys. Lett. B262 (1991) 477;

[19] H.E. Haber and R. Hempfling, Phys.Rev.Lett. 66 (1991) 1815; R. Barbieri, M. Frigeni and M. Caravaglios Phys. Lett. B258 (1991) 167; Y. Okada, M. Yamaguchi and T. Yanagida, Porg. Theor. Phys. 85 (1991) 1;

[20] see also H.E. Haber, "Higgs Boson Masses and Couplings in the Minimal Supersymmetric Model" and references therein, (hep-ph/9707213) to appear in Perspectives on Higgs Physics II Gordon L. Kane editor;

[21] V. Barger, J.L. Hewett and R.J.N. Phillips, Phys. Rev. D41 (1990) 3421; B. Schrempp and M, Wimmer, Prog. Part. Nucl. Phys. 37 (1996) 1;

[22] M. Sher Phys. Rep. 179, Nos $5 \& 6$ (1989) 273;

[23] R. Fukuda, T. Kugo, Phys. Rev. D 13 (1976) 3469; I.J.R. Aitchison, C.M. Fraser, Ann. Phys. 156 (1984) 1;

[24] see for instance, G. L. Kane "Tests and implications of increasing evidence for superpartners", Invited talk at XXXII Rencontres de Moriond, Les Arcs, March 1997; hep-ph/9705382; 


\section{Figure Captions}

Fig.1: The typical behaviour of $t_{ \pm}$versus $\zeta \equiv \frac{\tilde{\alpha} v_{1} v_{2}}{X_{m_{3}}^{2}}$ and in terms of the sign of $\tilde{\alpha}$.

Fig.2: An illustration of the typical dependence of the effective potential on the gaugefixing change. Extremal points have the same value for any $\xi_{1}, \xi_{2}, \ldots$. However, the curve $\xi$ leading to a gauge-fixing dependent minimum at $M^{\prime}$ is not allowed.

Fig.3: The $t_{1}$ solution of the stationarity equation for $\tan \beta$ as a function of the renormalization scale $\mu_{R}$. The bounds $\sqrt{t_{ \pm}}$are also shown to illustrate the rejection of $t_{1}$.

Fig.4: Same as Fig.3 for the non-rejected solution $t_{2}$. The tree-level value $\tan \beta_{0}$, the

relevant bounds $T_{+}$and $\sqrt{t_{+}}$and the scales at which the potential is unbounded from below are also shown.

Fig.5: Sensitivity of the tree-level and one-loop vev's to the renormalization scale. The one-loop vev's have an improved behaviour in the vicinity of and below the UFB scale. 


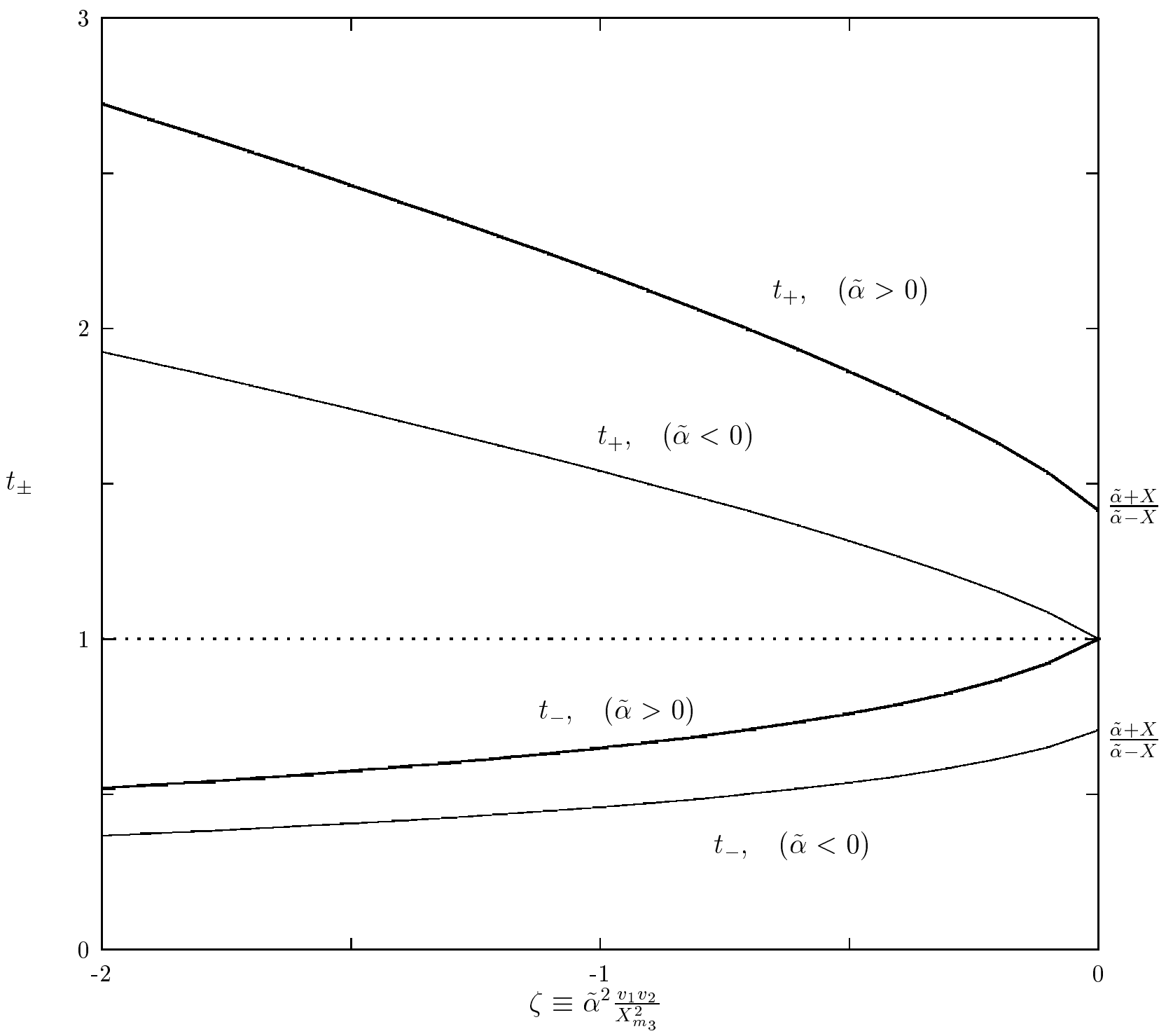

Fig.1 


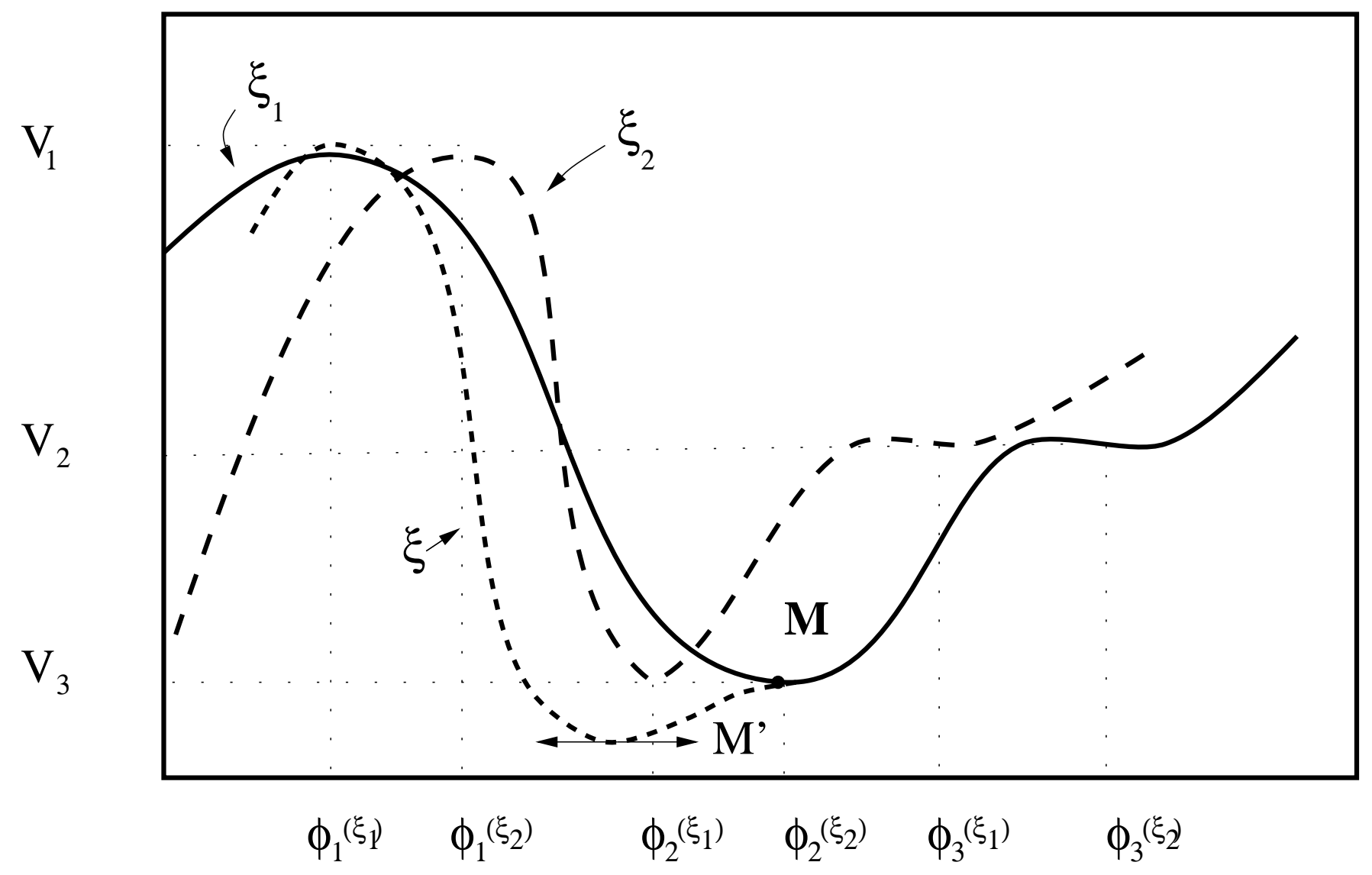

Fig.2 


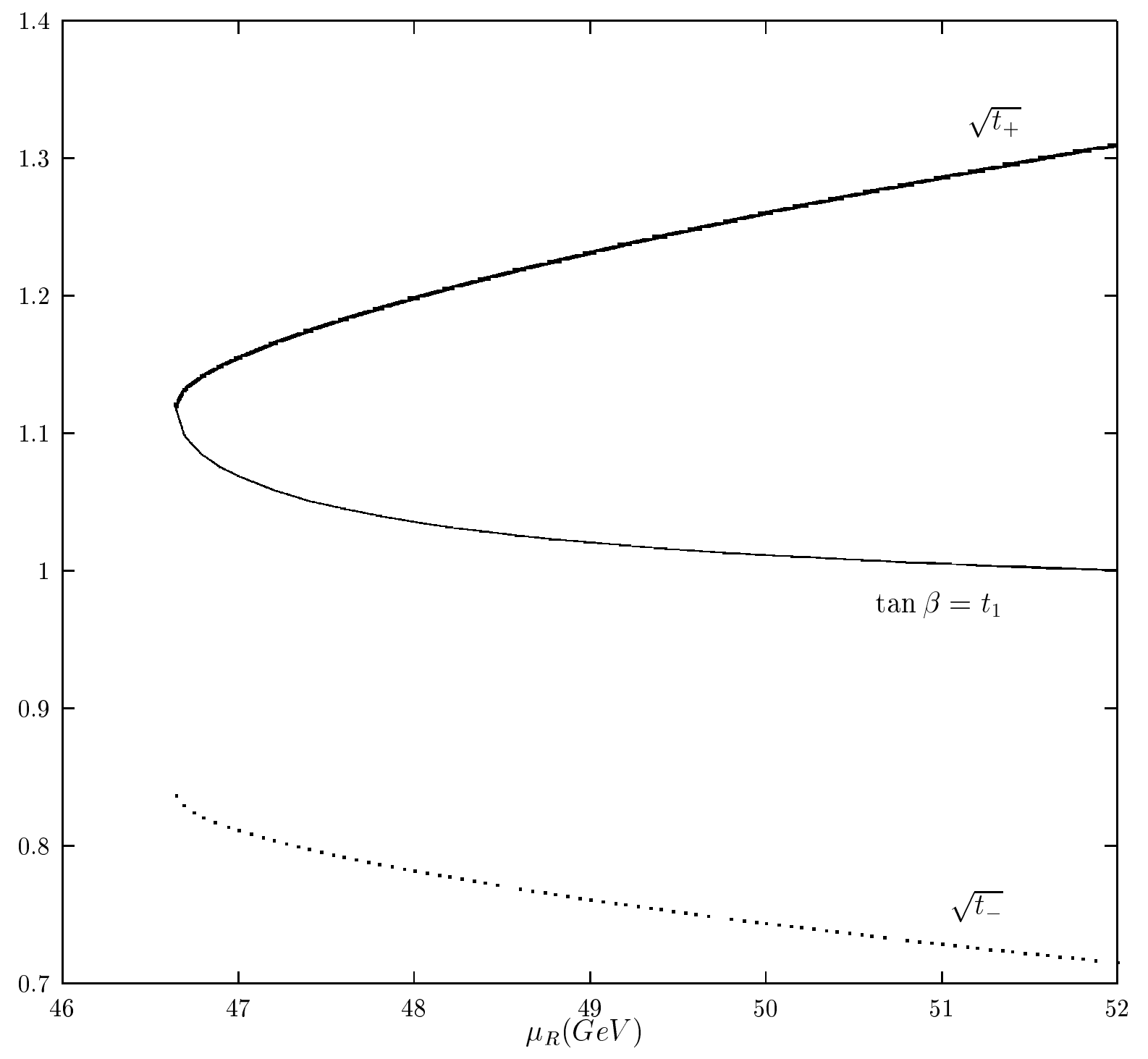

Fig.3 


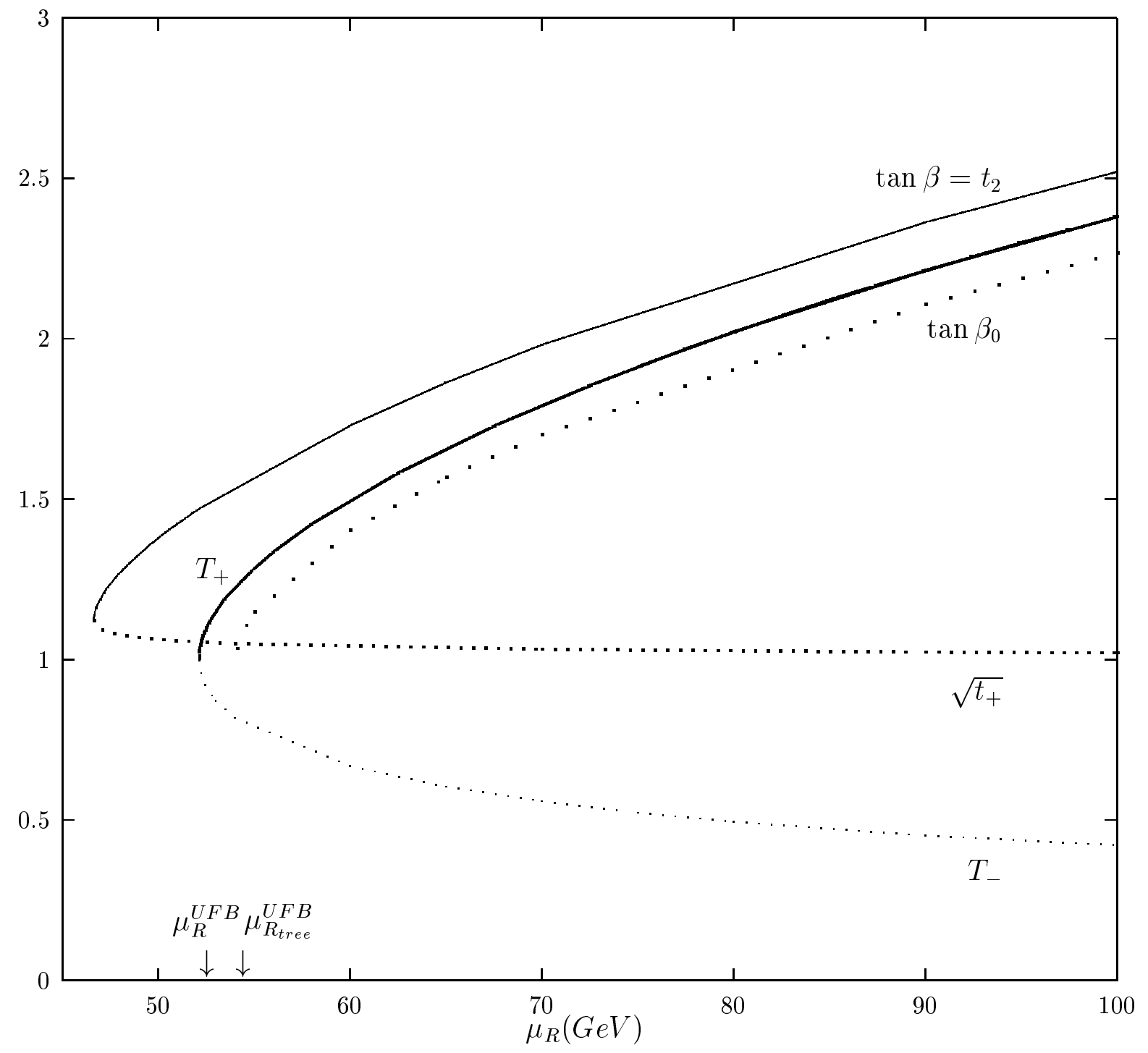

Fig.4 


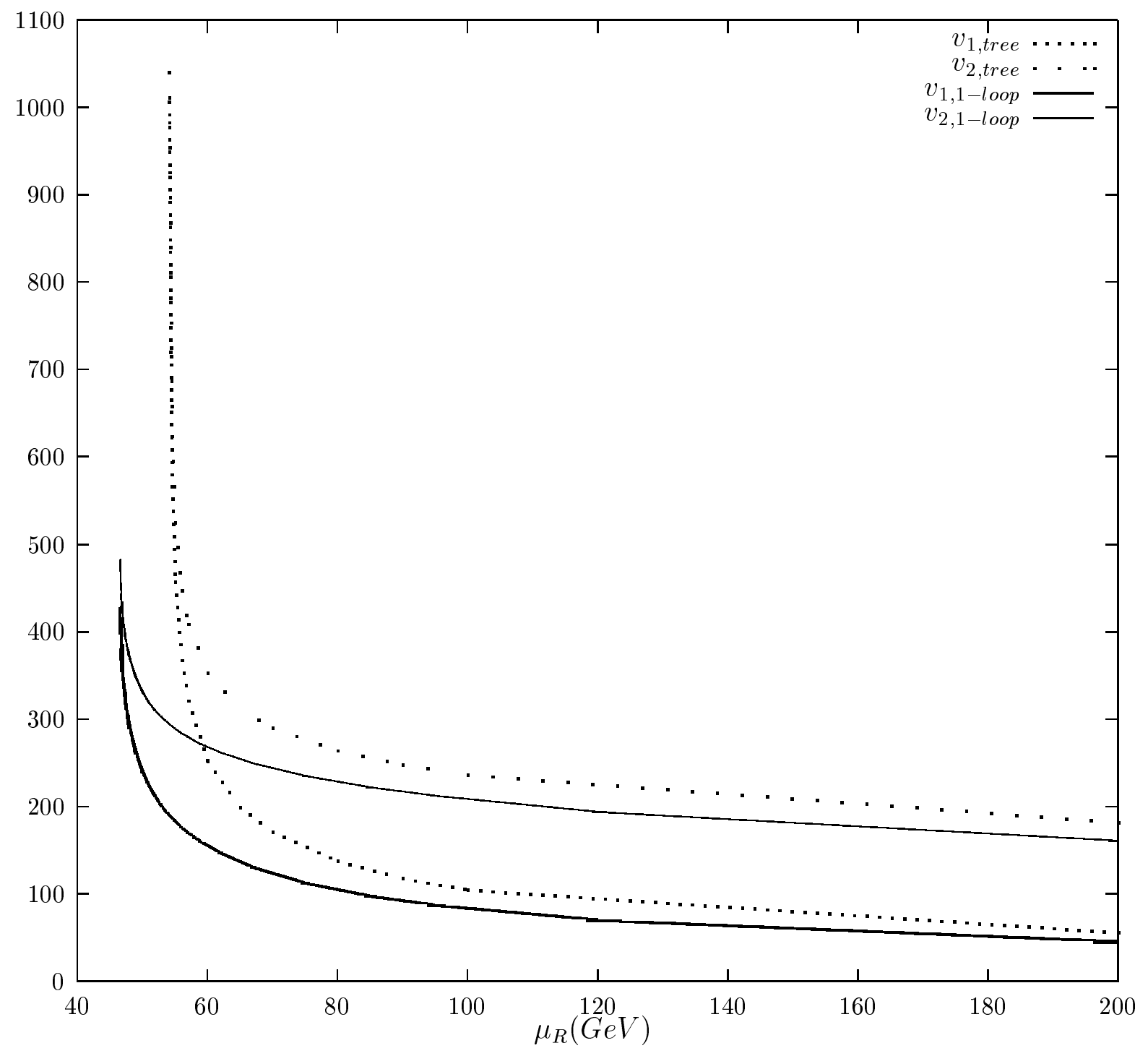

Fig.5 